\title{
Gabor Analysis in Weighted Amalgam Spaces
}

\author{
Karlheinz Gröchenig \\ Department of Mathematics \\ The University of Connecticut \\ Storrs, Connecticut 06269-3009 USA \\ groch@math. uconn. edu \\ Christopher Heil \\ School of Mathematics \\ Georgia Institute of Technology \\ Atlanta, Georgia 30332-0160 USA \\ heil@math.gatech.edu \\ Kasso Okoudjou \\ School of Mathematics \\ Georgia Institute of Technology \\ Atlanta, Georgia 30332-0160 USA \\ okoudjou@math.gatech.edu
}

\begin{abstract}
Gabor frames $\left\{e^{2 \pi i n \beta \cdot x} g(x-k \alpha)\right\}_{n, k \in \mathbb{Z}^{d}}$ provide series representations not only of functions in $L^{2}\left(\mathbb{R}^{d}\right)$ but of the entire range of spaces $M_{\nu}^{p, q}$ known as the modulation spaces. Membership of a function or distribution $f$ in the modulation space is characterized by a sequence-space norm of the Gabor coefficients of $f$ depending only on the magnitudes of those coefficients, and the Gabor series representation of $f$ converges unconditionally in the norm of the modulation space. This paper shows that Gabor expansions also converge in the entire range of amalgam spaces $W\left(L^{p}, L_{\nu}^{q}\right)$, which are not modulation spaces in general but, along with the modulation spaces, play important roles in time-frequency analysis and sampling theory. It is shown that membership of a function or distribution in the amalgam space is characterized by an appropriate
\end{abstract}


sequence space norm of the Gabor coefficients. However, this sequence space norm depends on the phase of the Gabor coefficients as well as their magnitudes, and the Gabor expansions converge conditionally in general. Additionally, some converse results providing necessary conditions on $g$ are obtained.

Key words and phrases: Amalgam spaces, frames, Gabor expansions, Gabor frames, modulation spaces, phase-space, sampling, time-frequency analysis, Walnut representation

2000 AMS Mathematics Subject Classification: 42C15, 42C20, $46 \mathrm{~B} 15$

\section{Introduction}

A Gabor frame $\mathcal{G}(g, \alpha, \beta)=\left\{e^{2 \pi i \beta n \cdot x} g(x-\alpha k)\right\}_{k, n \in \mathbb{Z}^{d}}$ for $L^{2}\left(\mathbb{R}^{d}\right)$ provides basis-like series representations of functions in $L^{2}$, with unconditional convergence of the series. However, unless the frame is a Riesz basis (and hence, by the Balian-Low theorem has poor time-frequency localization), these representations will not be unique. Still, a canonical and computable representation exists, and Gabor frames have found a wide variety of applications in mathematics, science, and engineering [9]. An important fact is that Gabor frames provide much more than just a means to recognize square-integrability of functions. If the window function $g$ is reasonably well-localized in time and frequency, then Gabor frame expansions are valid not only in $L^{2}$ but in an entire range of associated spaces $M_{\nu}^{p, q}$ known as the modulation spaces. The frame expansions converge unconditionally in the norm of those spaces, and membership of a tempered distribution in $M_{\nu}^{p, q}$ is characterized by membership of its sequence of Gabor coefficients in a weighted sequence space $\ell_{\tilde{\nu}}^{p, q}$. We refer to [25] for a recent detailed development of time-frequency analysis and modulation spaces.

Some results on Gabor analysis outside of the modulation spaces were obtained by Walnut in [34]. In particular, he introduced what is now known as the Walnut representation of the frame operator, and considered the boundedness of the frame operator on $L^{p}$. Recently, it was independently observed in [23] and [26] that Gabor expansions actually converge in $L^{p}\left(\mathbb{R}^{d}\right)$ when $1<p<\infty$. Since $L^{p}$ is not a modulation space when $p \neq 2$, it was known that Gabor expansions could not converge unconditionally in $L^{p}$. However, the fact that they converge at all was a surprise. 
In this paper we consider a much larger class of spaces than the $L^{p}$ spaces, namely, we consider the weighted amalgam spaces $W\left(L^{p}, L_{\nu}^{q}\right)$. These spaces amalgamate a local criteria for membership with a global criteria. They are the "right" spaces for a wide range of applications, and in particular play important roles in recent developments in timefrequency analysis [14], [16], [25] and in sampling theory [15], [1], [2], [3], [4], [19]. We will show that not only do Gabor expansions converge for the special case $L^{p}=W\left(L^{p}, L^{p}\right)$, but that they converge in the entire range of weighted amalgam spaces. Moreover, membership in the amalgam space is characterized by membership of the Gabor coefficients in an appropriate sequence space. In the course of obtaining these results, we prove several results of independent interest on the behavior of the analysis and synthesis operators associated with the Gabor frame, and on the Walnut representation, which is an extremely useful tool in Gabor frame theory. Moreover, we include the cases $p=1, \infty$ or $q=1, \infty$ in our consideration. In particular, we show that Gabor expansions exist even in $L^{1}$ and in a weak sense in $L^{\infty}$, given the right interpretation of "expansion." These results significantly extend the results in [26]. Additionally, we obtain some necessary conditions on the window $g$, extending weaker necessary conditions obtained by Balan in [5] for the particular case $W\left(L^{2}, L^{\infty}\right)$.

Amalgam space techniques play an important role in the formulation and proofs of sampling theorems. Specifically, we will prove the boundedness of the sampling operator on amalgam spaces. While our main focus will be on the Gabor analysis of amalgam spaces, we are convinced that our results on Gabor series are relevant for sampling problems per se, and we plan to exploit this connection in our future work.

Our paper is organized as follows. Following some basic notation in Section 2, we provide in Section 3 some background on Gabor expansions in $L^{2}$ and the modulation spaces. These set the stage for the precise statement of our main results in Section 4. Proofs of these results are given in Section 5.

\section{Notation}

\subsection{General notation}

In addition to the basic definitions and notation of [25], we use the fol- 
lowing notation. $Q_{\alpha}$ denotes the cube $Q_{\alpha}=[0, \alpha)^{d}$. The characteristic function of a measurable set $E$ is $\chi_{E}$. Translation and modulation of a function $f$ with domain $\mathbb{R}^{d}$ are defined, respectively, by

$$
T_{x} f(t)=f(t-x) \quad \text { and } \quad M_{y} f(t)=e^{2 \pi i y \cdot t} f(t) .
$$

The Fourier transform of $f \in L^{1}\left(\mathbb{R}^{d}\right)$ is

$$
\hat{f}(\omega)=\mathcal{F} f(\omega)=\int_{\mathbb{R}^{d}} f(t) e^{-2 \pi i t \cdot \omega} d t, \quad \omega \in \mathbb{R}^{d} .
$$

The Short-Time Fourier Transform (STFT) of a function $f$ with respect to a window $g$ is

$$
V_{g} f(x, y)=\left\langle f, M_{y} T_{x} g\right\rangle=\int_{\mathbb{R}^{d}} e^{-2 \pi i y \cdot t} \overline{g(t-x)} f(t) d t,
$$

whenever the integral makes sense. Analogously to the Fourier transform, the STFT extends in a distributional sense to $f, g$ in the space of tempered distributions $\mathcal{S}^{\prime}$, cf. [20, Prop. 1.42].

Given a strictly positive function $w$ on $\mathbb{R}^{d}$, the space $L_{w}^{p}$ is defined by the norm

$$
\|f\|_{L_{w}^{p}}=\|f w\|_{p}=\left(\int_{\mathbb{R}^{d}}|f(x)|^{p} w(x)^{p} d x\right)^{1 / p}
$$

with the usual adjustment if $p=\infty$. Here and in other definitions, if $w \equiv 1$ then we omit writing it.

Given $E \subset \mathbb{R}^{d}$, we use the shorthand

$$
\|f\|_{p, E}=\left\|f \cdot \chi_{E}\right\|_{L^{p}}=\left(\int_{E}|f(x)|^{p} d x\right)^{1 / p} .
$$

\subsection{Weight functions}

Throughout this paper, $\omega$ will denote a submultiplicative weight function, i.e., $\omega$ is positive, symmetric, and continuous, and satisfies

$$
\forall x, y \in \mathbb{R}^{d}, \quad \omega(x+y) \leq \omega(x) \omega(y) .
$$

The prototypical example of a submultiplicative weight is the polynomially-growing function $\omega(x)=(1+|x|)^{s}$, where $s>0$. We also 
consider weight functions defined on $\mathbb{R}^{2 d}$ by making the obvious changes in the definition.

Throughout this paper, $\nu$ will denote an $\omega$-moderate function, i.e., $\nu$ is positive, continuous, and symmetric, and there exists a constant $C_{\nu}>0$ such that

$$
\forall x, y \in \mathbb{R}^{d}, \quad \nu(x+y) \leq C_{\nu} \omega(x) \nu(y) .
$$

For example, $\nu(x)=(1+|x|)^{t}$ is moderate with respect to $\omega(x)=$ $(1+|x|)^{s}$ exactly for $|t| \leq s$.

If $\nu$ is $\omega$-moderate, then by manipulating (1) we see that

$$
\frac{1}{\nu(x+y)} \leq C_{\nu} \omega(x) \frac{1}{\nu(y)}
$$

so $1 / \nu$ is also $\omega$-moderate (with the same constant). Thus, the class of $\omega$ moderate weights is closed under reciprocals, and consequently the class of spaces $L_{\nu}^{p}$ using $\omega$-moderate weights is closed under duality (with the usual exception for $p=\infty$ ). This would not be the case if we restricted only to submultiplicative weights. The following lemma provides an important additional motivation for considering moderate weights, namely that $L_{\nu}^{p}$ is translation-invariant exactly for moderate weights [11].

Lemma 2.1. Let $\omega$ be a submultiplicative weight on $\mathbb{R}^{d}$, and fix $1 \leq p \leq$ $\infty$. Then the following statements are equivalent.

a. $\nu$ is $\omega$-moderate.

b. $L_{\nu}^{p}$ is translation-invariant (i.e., for each $x \in \mathbb{R}^{d}, T_{x}$ is a continuous mapping of $L_{\nu}^{p}$ onto itself).

c. For each compact set $K \subset \mathbb{R}^{d}$, there exists a constant $C>0$ such that

$$
\forall y \in \mathbb{R}^{d}, \quad \sup _{t \in y+K} \nu(t) \leq C \inf _{t \in y+K} \nu(t) .
$$

Given an $\omega$-moderate weight $\nu$ on $\mathbb{R}^{d}$, we will often use the notation $\tilde{\nu}$ to denote the weight on $\mathbb{Z}^{d}$ defined by $\tilde{\nu}(k)=\nu(\alpha k)$, and for a weight $\nu$ on $\mathbb{R}^{2 d}$ we define $\tilde{\nu}(k, n)=\nu(\alpha k, \beta n)$. 


\subsection{Amalgam spaces}

Given an $\omega$-moderate weight $\nu$ on $\mathbb{R}^{d}$ and given $1 \leq p, q \leq \infty$, the weighted amalgam space $W\left(L^{p}, L_{\nu}^{q}\right)$ is the Banach space of all measurable functions on $\mathbb{R}^{d}$ for which the norm

$$
\|f\|_{W\left(L^{p}, L_{\nu}^{q}\right)}=\left(\sum_{k \in \mathbb{Z}^{d}}\left\|f \cdot T_{\alpha k} \chi_{Q_{\alpha}}\right\|_{p}^{q} \nu(\alpha k)^{q}\right)^{1 / q}
$$

is finite, with the usual adjustment if $q=\infty$.

The first use of amalgam spaces was by Wiener, who introduced the spaces $W\left(L^{1}, L^{2}\right)$ and $W\left(L^{2}, L^{1}\right)$ in [35] and $W\left(L^{\infty}, L^{1}\right)$ and $W\left(L^{1}, L^{\infty}\right)$ in [36], [37], in connection with his development of the theory of generalized harmonic analysis. The space $W\left(L^{\infty}, L^{1}\right)$ is sometimes called the Wiener algebra, cf. [32]. It was shown in [34] that $W\left(L^{\infty}, L^{1}\right)$ is a convenient and general class of windows for Gabor analysis within $L^{2}$.

In a series of papers beginning with [12], [13], Feichtinger developed a comprehensive theory of amalgam spaces on locally compact groups which allows a wide range of spaces of functions or distributions to be used as local or global components in the amalgam, not just spaces such as $L^{p}$ or $L_{\nu}^{q}$ that are defined solely in terms of integrability conditions. See [14], [15], [16] for discussions of amalgam spaces and their applications. For an introduction to amalgams, concentrating on the weighted amalgams $W\left(L^{p}, L_{\nu}^{q}\right)$ but providing an introduction to the general Feichtinger theory and containing background and references, we refer to [28]. Additionally, the review of Fournier and Stewart [21] is a useful introduction to the amalgams $W\left(L^{p}, L^{q}\right)$ in the setting of locally compact abelian groups.

Since any cube $Q_{\alpha}$ in $\mathbb{R}^{d}$ can be covered by a finite number of translates of a cube $Q_{\beta}$, the space $W\left(L^{p}, L_{\nu}^{q}\right)$ is independent of the value of $\alpha$ used in (2) in the sense that each different choice of $\alpha$ yields an equivalent norm for $W\left(L^{p}, L_{\nu}^{q}\right)$. A wide variety of other equivalent norms is provided by the Feichtinger amalgam theory. In particular, we refer to [28] for an exposition of the "continuous" norms on the amalgam spaces, which provide a much clearer motivation than (2) of why $W\left(L^{p}, L_{\nu}^{q}\right)$ should be viewed as an amalgamation of a local $L^{p}$ with a global $L_{\nu}^{q}$, rather than merely a disjoint piecing together of local $L^{p}$ components.

For each $\omega$-moderate weight $\nu$, we have the following inclusion rela- 
tions: if $p_{1} \geq p_{2}, q_{1} \leq q_{2}$, then

$$
W\left(L^{p_{1}}, L_{\omega}^{q_{1}}\right) \subset W\left(L^{p_{1}}, L_{\nu}^{q_{1}}\right) \subset W\left(L^{p_{2}}, L_{\nu}^{q_{2}}\right) \subset W\left(L^{p_{2}}, L_{1 / \omega}^{q_{2}}\right) .
$$

In particular, the inclusions $W\left(L^{\infty}, L_{\omega}^{1}\right) \subset W\left(L^{p}, L_{\nu}^{q}\right) \subset W\left(L^{1}, L_{1 / \omega}^{\infty}\right)$ hold for all $1 \leq p, q \leq \infty$ and all $\omega$-moderate weights $\nu$. In this sense $W\left(L^{\infty}, L_{\omega}^{1}\right)$ is the smallest and $W\left(L^{1}, L_{1 / \omega}^{\infty}\right)$ is the largest amalgam space in the class of amalgam spaces with $\omega$-moderate weight functions.

For $p, q<\infty$, the Schwartz class $\mathcal{S}$ and the space of functions with compact support are dense in $W\left(L^{p}, L_{\nu}^{q}\right)$.

\subsection{Amalgam Spaces and Sampling Theory}

To highlight the important role of amalgam spaces in sampling theory, we show how amalgam spaces arise in the rigorous definition of the sampling operator. For more details on amalgam space techniques in sampling theory, we refer to the pioneering work of Feichtinger [14], [15] and the recent survey [4].

A discrete set $X=\left\{x_{j}\right\}_{j \in J} \subset \mathbb{R}^{d}$ is called separated if $\inf _{j \neq k} \mid x_{j}-$ $x_{k} \mid>0$, and relatively separated if $X$ is a finite union of separated sets. The sampling operator $S_{X} f$ is defined as the restriction of $f$ to $X$, i.e., it maps a function $f$ to the sequence $S_{X} f=\left\{f\left(x_{j}\right)\right\}_{j \in J}$. In general, $S_{X} f$ need not even be defined, therefore we must impose conditions on $f$ in order that the sampling operator be a well-defined object. Here amalgam spaces are very useful.

Theorem 2.2. $\quad$ a. If $X$ is a relatively separated set, then the sampling operator is a bounded operator from $W\left(L^{\infty}, L_{\nu}^{q}\right) \cap C$ to $\ell_{\tilde{\nu}}^{q}(J)$, and thus

$$
\left(\sum_{j \in J}\left|f\left(x_{j}\right)\right|^{q} \nu\left(x_{j}\right)^{q}\right)^{1 / q} \leq C\|f\|_{W\left(L^{\infty}, L_{\nu}^{q}\right)} .
$$

b. A continuous function $f$ belongs to $W\left(L^{\infty}, L_{\nu}^{q}\right)$ if and only if $S_{X} f \in \ell_{\tilde{\nu}}^{q}(J)$ for all relatively separated sets $X \subset \mathbb{R}^{d}$.

Proof. For a proof of part a, we refer to Prop. 11.1.4 (and the remark following) in [25], and to [4, Thm. 3.1].

(b) If $f$ is a continuous function in $W\left(L^{\infty}, L_{\nu}^{q}\right)$, then $S_{X} f \in \ell_{\tilde{\nu}}^{q}(J)$ by part a. 
Conversely, assume that $f$ is continuous and that $S_{X} f \in \ell_{\tilde{\nu}}^{q}(J)$ for all relatively separated sets $X \subset \mathbb{R}^{d}$. For each $k \in \mathbb{Z}^{d}$, choose $x_{k} \in \alpha k+Q_{\alpha}$ with the property that $\left|f\left(x_{k}\right)\right|=\left\|f \cdot T_{\alpha k} \chi_{Q_{\alpha}}\right\|_{\infty}$. Then the set $\left\{x_{k}\right\}_{k \in \mathbb{Z}^{d}}$ is relatively separated. Using Lemma 2.1(c) and our assumption, we find that

$$
\begin{aligned}
\left\|S_{X} f\right\|_{\ell_{\bar{\nu}}^{q}} & =\left(\sum_{k \in \mathbb{Z}^{d}}\left|f\left(x_{k}\right)\right|^{q} \nu\left(x_{k}\right)^{q}\right)^{1 / q} \\
& \geq C\left(\sum_{k \in \mathbb{Z}^{d}}\left\|f \cdot T_{\alpha k} \chi_{Q_{\alpha}}\right\|_{\infty}^{q} \nu(\alpha k)^{q}\right)^{1 / q} \\
& =C\|f\|_{W\left(L^{\infty}, L_{\nu}^{q}\right)} .
\end{aligned}
$$

Strictly speaking, any real sampling device takes only local averages $\left\langle f, \psi_{j}\right\rangle$ instead of the exact point evaluations $f\left(x_{j}\right)$, cf. [1]. Here, the $\psi_{j}$ 's form a collection of averaging functions associated to the sampling set $X$, and they are assumed to satisfy the following natural properties:

$$
\begin{array}{cc}
\operatorname{supp}\left(\psi_{j}\right) \subset x_{j}+Q_{\alpha} & \text { for some } \alpha>0, \\
\int_{\mathbb{R}^{d}} \psi_{j}(x) d x=1, & \\
\sup _{j \in J}\left\|\psi_{j}\right\|_{p^{\prime}}=M<\infty & \text { for some } 1 \leq p \leq \infty .
\end{array}
$$

A more realistic sampling model will then replace the sampling operator $S_{X}$ by the averaged sampling operator $A_{X, \Psi}$ defined by $A_{X, \Psi} f=$ $\left\{\left\langle f, \psi_{j}\right\rangle\right\}_{j \in J}$. As above, $A_{X, \Psi}$ is well-defined only on certain function spaces, precisely some amalgam spaces, as is shown in the following statement.

Proposition 2.3. If $X \subset \mathbb{R}^{d}$ is a relatively separated set and $\Psi=$ $\left\{\psi_{j}\right\}_{j \in J}$ is a set of averaging functions satisfying (3)-(5), then $A_{X, \Psi}$ is a bounded operator from $W\left(L^{p}, L_{\nu}^{q}\right)$ to $\ell_{\tilde{\nu}}^{q}(J)$.

Proof. First we note that

$$
\max _{k \in \mathbb{Z}^{d}} \#\left\{j \in J: x_{j} \in \alpha k+Q_{\alpha}\right\}=N_{0}<\infty,
$$

because $X$ is relatively separated. Now, if $x_{j} \in \alpha k+Q_{\alpha}$, then $\operatorname{supp}\left(\psi_{j}\right) \subset$ $x_{j}+Q_{\alpha} \subset \alpha k+Q_{2 \alpha}$, and therefore

$$
\left|\left\langle f, \psi_{j}\right\rangle\right| \leq\left\|f \cdot T_{\alpha k} \chi_{Q_{2 \alpha}}\right\|_{p}\left\|\psi_{j}\right\|_{p^{\prime}}
$$


Using (5), (6), and Lemma 2.1, we then obtain that

$$
\begin{aligned}
\left\|A_{X, \Psi} f\right\|_{\ell_{\tilde{\nu}}^{q}}^{q} & =\sum_{j \in J}\left|\left\langle f, \psi_{j}\right\rangle\right|^{q} \nu\left(x_{j}\right)^{q} \\
& =\sum_{k \in \mathbb{Z}^{d} x_{j} \in \alpha k+Q_{\alpha}} \sum_{\left.\left\langle f, \psi_{j}\right\rangle\right|^{q} \nu\left(x_{j}\right)^{q}} \\
& \leq N_{0} M C \sum_{k \in \mathbb{Z}^{d}}\left\|f \cdot T_{\alpha k} \chi_{Q_{2 \alpha}}\right\|_{p}^{q} \nu(\alpha k)^{q} \\
& \leq C_{1}\|f\|_{W\left(L^{p}, L_{\nu}^{q}\right)}^{q},
\end{aligned}
$$

as desired.

\subsection{Duality and convergence}

We will need to be precise about the meaning of convergence of series. For general references we refer to the text of Singer [33], and for references on Banach function spaces we refer to the text of Bennett and Sharpley [8].

The following lemma characterizing unconditional convergence will be useful.

Lemma 2.4. Let $X$ be a Banach space with dual space $X^{*}$, and let $f_{k} \in X$ for $k \in J$. Then the following statements are equivalent.

a. $\sum_{k \in J} f_{k}$ converges unconditionally in $X$, i.e., it converges with respect to every ordering of the index set $J$.

b. There exists $f \in X$ such that for each $\varepsilon>0$, there exists a finite $F_{0} \subset J$ such that

$$
\forall \text { finite } F \supset F_{0}, \quad\left\|f-\sum_{k \in F} f_{k}\right\|_{X}<\varepsilon .
$$

c. For every $\varepsilon>0$, there exists a finite $F_{0} \subset J$ such that

$$
\forall \text { finite } F \supset F_{0}, \quad \sup \left\{\sum_{k \notin F}\left|\left\langle f_{k}, h\right\rangle\right|: h \in X^{*},\|h\|_{X^{*}}=1\right\}<\varepsilon .
$$

Now let $X$ be a Banach function space in the sense of [8]. In particular, this includes the amalgam spaces $W\left(L^{p}, L_{\nu}^{q}\right)$. The Köthe dual 
(or associated space, as it is called in [8]), is the space $\tilde{X}$ consisting of all measurable functions $h$ such that $f h \in L^{1}$ for each $f \in X$. By [8, Thm. 1.2.9], $\tilde{X}$ is a closed, norm-fundamental subspace of $X^{*}$, so in particular,

$$
\forall f \in X, \quad\|f\|_{X}=\sup \left\{|\langle f, h\rangle|: h \in \tilde{X},\|h\|_{\tilde{X}}=1\right\} .
$$

By $[8$, Cor. 1.5.3], $X$ is complete in the $\sigma(X, \tilde{X})$ topology, i.e., the weak topology on $X$ generated by $\tilde{X}$. In particular, a series $\sum_{k \in J} f_{k}$ converges in the $\sigma(X, \tilde{X})$ topology if $\sum_{k \in J}\left\langle f_{k}, h\right\rangle$ converges for each $h \in \tilde{X}$. It converges unconditionally in that topology if the convergence is independent of the ordering of $J$, and since the terms $\left\langle f_{k}, h\right\rangle$ are scalars, this occurs if and only if

$$
\forall h \in \tilde{X}, \quad \sum_{k \in J}\left|\left\langle f_{k}, h\right\rangle\right|<\infty .
$$

The dual and Köthe dual of the amalgam spaces are given in the next lemma.

Lemma 2.5. Let $\nu$ be an $\omega$-moderate weight.

a. For $1 \leq p, q<\infty$, the dual space of $W\left(L^{p}, L_{\nu}^{q}\right)$ is $W\left(L^{p^{\prime}}, L_{1 / \nu}^{q^{\prime}}\right)$.

b. For $1 \leq p, q \leq \infty$, the Köthe dual of $W\left(L^{p}, L_{\nu}^{q}\right)$ is $W\left(L^{p^{\prime}}, L_{1 / \nu}^{q^{\prime}}\right)$.

\section{Background: Gabor Expansions in $L^{2}$ and the Modulation Spaces}

\subsection{Gabor frames in $L^{2}$}

Given a window function $g \in L^{2}\left(\mathbb{R}^{d}\right)$ and given $\alpha, \beta>0$, we say that

$$
\mathcal{G}(g, \alpha, \beta)=\left\{M_{\beta n} T_{\alpha k} g\right\}_{k, n \in \mathbb{Z}^{d}}=\left\{e^{2 \pi i \beta n \cdot x} g(x-\alpha k)\right\}_{k, n \in \mathbb{Z}^{d}}
$$

is a Gabor frame for $L^{2}\left(\mathbb{R}^{d}\right)$ if there exist constants $A, B>0$ (called frame bounds) such that

$$
\forall f \in L^{2}\left(\mathbb{R}^{d}\right), \quad A\|f\|_{L^{2}}^{2} \leq \sum_{k, n \in \mathbb{Z}^{d}}\left|\left\langle f, M_{\beta n} T_{\alpha k} g\right\rangle\right|^{2} \leq B\|f\|_{L^{2}}^{2} .
$$

The basic properties of Gabor frames are laid out in the following result; we refer to [9], [25], or [29] for more extensive treatments of frames and Gabor frames. 
Theorem 3.1. Let $\mathcal{G}(g, \alpha, \beta)$ be a Gabor frame for $L^{2}\left(\mathbb{R}^{d}\right)$ with frame bounds $A, B$. Then the following statements hold.

a. The analysis operator $C_{g} f=\left(\left\langle f, M_{\beta n} T_{\alpha k} g\right\rangle\right)_{k, n \in \mathbb{Z}^{d}}$ is a bounded mapping $C_{g}: L^{2} \rightarrow \ell^{2}$, and we have the norm equivalence $\|f\|_{2} \asymp$ $\left\|C_{g} f\right\|_{\ell^{2}}$.

b. The synthesis operator $R_{g} c=\sum_{k, n \in \mathbb{Z}^{d}} c_{k n} M_{\beta n} T_{\alpha k} g$ is a bounded mapping $R_{g}: \ell^{2} \rightarrow L^{2}$. The series defining $R_{g} c$ converges unconditionally in $L^{2}$ for every $c \in \ell^{2}$.

c. $R_{g}=C_{g}^{*}$, and the frame operator $S_{g}=R_{g} C_{g}: L^{2} \rightarrow L^{2}$ is strictly positive.

d. The dual window $\gamma=S_{g}^{-1} g$ generates a Gabor frame $\mathcal{G}(\gamma, \alpha, \beta)$ for $L^{2}\left(\mathbb{R}^{d}\right)$ with frame bounds $1 / B, 1 / A$.

e. $R_{\gamma} C_{g}=I$ on $L^{2}\left(\mathbb{R}^{d}\right)$, i.e., we have the Gabor expansions

$$
f=R_{\gamma} C_{g} f=\sum_{k, n \in \mathbb{Z}^{d}}\left\langle f, M_{\beta n} T_{\alpha k} \gamma\right\rangle M_{\beta n} T_{\alpha k} g
$$

for $f \in L^{2}\left(\mathbb{R}^{d}\right)$, with unconditional convergence of the series.

In brief, if $\mathcal{G}(g, \alpha, \beta)$ is a frame for $L^{2}\left(\mathbb{R}^{d}\right)$ then the $\ell^{2}$-norm of the sequence of Gabor coefficients $\left(\left\langle f, M_{\beta n} T_{\alpha k} g\right\rangle\right)_{k, n \in \mathbb{Z}^{d}}$ is an equivalent norm for $L^{2}$, and the Gabor expansions given by $(7)$ hold in $L^{2}$. Moreover, for our purposes it is important to note that once the analysis and synthesis operators are defined, the statement "Gabor expansions converge in $L^{2}$ " is equivalent to the statement that the identity operator on $L^{2}$ factorizes as $I=R_{\gamma} C_{g}$.

In all these statements, and throughout this paper, the roles of $g$ and $\gamma$ may be interchanged.

\subsection{Gabor frames in the modulation spaces}

Under stronger assumptions on $g$, the expansions in (7) are valid not only in $L^{2}$ but in the entire class of function spaces known as the modulation spaces. For detailed discussion of these spaces we refer to [17], [25]. The appropriate window class in this setting is the Feichtinger algebra

$$
M_{\omega}^{1}=\left\{f \in \mathcal{S}^{\prime}\left(\mathbb{R}^{d}\right): V_{f} f \in L_{\omega}^{1}\left(\mathbb{R}^{2 d}\right)\right\} .
$$


where $\omega$ is a submultiplicative weight on $\mathbb{R}^{2 d}$ with polynomial growth (see [25, Sec. 11.4] for a discussion of the details required in dealing with more general weights). The Schwartz class $\mathcal{S}$ is dense in $M_{\omega}^{1}$. Since $\omega \geq 1$, we have $M_{\omega}^{1} \subset M^{1}$, and it can be shown that $M^{1}$ is contained in the Wiener algebra $W\left(L^{\infty}, L^{1}\right)$.

Let $\nu$ be an $\omega$-moderate weight function on $\mathbb{R}^{2 d}$, and let $g \in M_{\omega}^{1}$ be fixed. Then the modulation space $M_{\nu}^{p, q}$ is the space of all tempered distributions $f \in \mathcal{S}^{\prime}\left(\mathbb{R}^{d}\right)$ for which the norm

$$
\|f\|_{M_{\nu}^{p, q}}=\left(\int_{\mathbb{R}^{d}}\left(\int_{\mathbb{R}^{d}}\left|V_{g} f(x, y)\right|^{p} \nu(x, y)^{p} d x\right)^{q / p} d y\right)^{1 / q}
$$

is finite. This definition is independent of the choice of $g \in M_{\omega}^{1}$ in the sense of equivalent norms. Further, $M_{\omega}^{1}=M_{\omega}^{1,1}$ (with equivalent norms). For $1 \leq p, q<\infty$, the dual of $M_{\nu}^{p, q}$ is $M_{1 / \nu}^{p^{\prime}, q^{\prime}}$. The space $M_{\nu}^{\infty, \infty}$ is a subspace of the tempered distributions, but has the advantage of being a Banach space. If $\nu(x, y)=\nu(x)=(1+|x|)^{s}$, then $M_{\nu}^{2,2}$ is the weighted $L^{2}$ space $L_{\nu}^{2}$. If $\nu(x, y)=\nu(y)=(1+|y|)^{s}$, then $M_{\nu}^{2,2}=H^{s}$, the standard Sobolev space. However, $L^{p}$ does not coincide with any modulation space when $p \neq 2[18]$.

The following result summarizes some basic facts on Gabor frames in the modulation spaces, cf. [25, Ch. 12]. The theorem is not stated in its weakest possible form; for example, the boundedness of the analysis and synthesis operators requires only the assumption $g \in M_{\omega}^{1}$, and does not require that $g$ generate a frame for $L^{2}$. The mixed-norm sequence space $\ell_{\tilde{\nu}}^{p, q}$ consists of all sequences $c=\left(c_{k n}\right)_{k, n \in \mathbb{Z}^{d}}$ such that

$$
\|c\|_{\ell_{\tilde{\nu}}^{p, q}}=\left(\sum_{n \in \mathbb{Z}^{d}}\left(\sum_{k \in \mathbb{Z}^{d}}\left|c_{k n}\right|^{p} \tilde{\nu}(k, n)^{p}\right)^{q / p}\right)^{1 / q}<\infty,
$$

where $\tilde{\nu}(k, n)=\nu(\alpha k, \beta n)$.

Theorem 3.2. Let $\nu$ be an $\omega$-moderate weight on $\mathbb{R}^{2 d}$, and let $1 \leq$ $p, q \leq \infty$. Let $g \in M_{\omega}^{1}$ be such that $\mathcal{G}(g, \alpha, \beta)$ is a Gabor frame for $L^{2}\left(\mathbb{R}^{d}\right)$. Then the following statements hold.

a. The analysis operator $C_{g} f=\left(\left\langle f, M_{\beta n} T_{\alpha k} g\right\rangle\right)_{k, n \in \mathbb{Z}^{d}}$ is a bounded mapping $C_{g}: M_{\nu}^{p, q} \rightarrow \ell_{\tilde{\nu}}^{p, q}$, and we have the norm equivalence

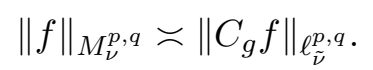


b. The synthesis operator $R_{g} c=\sum_{k, n \in \mathbb{Z}^{d}} c_{k n} M_{\beta n} T_{\alpha k} g$ is a bounded mapping $R_{g}: \ell_{\tilde{\nu}}^{p, q} \rightarrow M_{\nu}^{p, q}$. The series defining $R_{g} c$ converges unconditionally in the norm of $M_{\nu}^{p, q}$ for every $c \in \ell_{\tilde{\nu}}^{p, q}$ (weak* unconditionally in $M_{1 / \omega}^{\infty, \infty}$ if $p=\infty$ or $q=\infty$ ).

c. The frame operator $S_{g}=R_{g} C_{g}$ is a continuously invertible mapping of $M_{\nu}^{p, q}$ onto itself.

d. The dual window $\gamma=S_{g}^{-1} g$ lies in $M_{\omega}^{1}$.

e. $R_{\gamma} C_{g}=I$ on $M_{\nu}^{p, q}$.

f. A distribution $f \in M_{\nu}^{\infty, \infty}$ belongs to $M_{\nu}^{p, q}$ if and only if $C_{g} f \in \ell_{\tilde{\nu}}^{p, q}$. If $g \in \mathcal{S}$, then a tempered distribution $f \in \mathcal{S}^{\prime}\left(\mathbb{R}^{d}\right)$ belongs to $M_{\nu}^{p, q}$ if and only if $C_{g} f \in \ell_{\tilde{\nu}}^{p, q}$.

In brief, the $\ell_{\tilde{\nu}}^{p, q}$ norm of the Gabor coefficients $\left(\left\langle f, M_{\beta n} T_{\alpha k} g\right\rangle\right)_{k, n \in \mathbb{Z}^{d}}$ is an equivalent norm for $M_{\nu}^{p, q}$, and the Gabor expansions (7) are valid in $M_{\nu}^{p, q}$, with unconditional convergence of that series in the norm of $M_{\nu}^{p, q}$. Moreover, there is a strong statement made in part $f$ of Theorem 3.2 that is not usually observed in the standard list of Gabor frame properties in $L^{2}$ (Theorem 3.1), namely that $\left\|C_{g} f\right\|_{\ell_{\bar{\nu}}^{p, q}}$ is not only an equivalent norm for $M_{\nu}^{p, q}$, but membership of $f$ in the modulation space is characterized by membership of its sequence of Gabor coefficients $C_{g} f$ in $\ell_{\tilde{\nu}}^{p, q}$. In particular, only the magnitude of these coefficients is important in determining whether a given distribution lies in $M_{\nu}^{p, q}$.

The proof of Theorem 3.2 requires deep analysis. In particular, the invertibility of $S_{g}$ on $M_{\omega}^{1,1}$ for arbitrary values of $\alpha, \beta$ was only recently proved in [27].

In summary, once the analysis and synthesis operators have been correctly defined, the fact that Gabor expansions converge in the modulation spaces is simply the statement that the identity operator on $M_{\nu}^{p, q}$ factorizes as $I=R_{\gamma} C_{g}$.

Remark 3.3. For comparison with later results, let us rewrite the definition of the synthesis operator in several ways:

$$
\begin{aligned}
R_{g} c(x) & =\sum_{k, n \in \mathbb{Z}^{d}} c_{k n} M_{\beta n} T_{\alpha k} g(x) \\
& =\sum_{k \in \mathbb{Z}^{d}}\left(\sum_{n \in \mathbb{Z}^{d}} c_{k n} e^{2 \pi i \beta n \cdot x}\right) T_{\alpha k} g(x)
\end{aligned}
$$




$$
=\sum_{k \in \mathbb{Z}^{d}} m_{k}(x) T_{\alpha k} g(x)
$$

where $m_{k}(x)=\sum_{n \in \mathbb{Z}^{d}} c_{k n} e^{2 \pi i \beta n \cdot x}$ is a $1 / \beta$-periodic function. In the modulation spaces, the convergence of the double sum in (8) is unconditional, but when we turn to the amalgam spaces in Section 4, this will no longer be the case. A correct formulation of the meaning of the synthesis operator will then be essential, and the form of $R_{g} c$ given by (9) or especially (10) becomes the appropriate inspiration.

One potential point of confusion in comparing the statement of results for amalgam spaces with the modulation spaces is that although the amalgam spaces $W\left(L^{p}, L_{\nu}^{q}\right)$ and the modulation spaces $M_{\nu}^{p, q}$ both involve a weight, the weight serves different purposes and is defined on different spaces. For the modulation spaces, the weight is a joint timefrequency weight, and hence is defined on $\mathbb{R}^{2 d}$, while for the amalgams it is a weight on the global component of the amalgam, and hence is a weight on $\mathbb{R}^{d}$.

\section{Gabor Expansions in the Amalgam Spaces}

In this section we will state our main results precisely. In particular, we show that there is an analogue of Theorem 3.2 for the case of Gabor expansions in the weighted amalgam spaces. This is surprising, because the modulation spaces are the natural setting for Gabor analysis. And indeed, while Gabor expansions converge unconditionally in the modulation spaces, the convergence in the amalgam spaces is conditional in general and even the meaning of the term "expansion" must be handled appropriately. Throughout, we will use the notation $\tilde{\nu}(k)=\nu(\alpha k)$.

\subsection{Sequence spaces}

Before stating our results, we must define the sequence spaces that will be associated with Gabor expansions in the amalgam spaces. We begin by recalling that the Fourier transform of $f \in L^{1}\left(Q_{1 / \beta}\right)$ is the sequence $\hat{f}$ defined by

$$
\hat{f}(n)=\mathcal{F} f(n)=\beta^{d} \int_{Q_{1 / \beta}} f(t) e^{-2 \pi i \beta n \cdot t} d t, \quad n \in \mathbb{Z}^{d} .
$$


For $1 \leq p \leq \infty$, let $\mathcal{F} L^{p}\left(Q_{1 / \beta}\right)$ denote the image of $L^{p}\left(Q_{1 / \beta}\right)$ under the Fourier transform. Since Fourier coefficients are unique in $L^{p}$, if $c=$ $\left(c_{n}\right)_{n \in \mathbb{Z}^{d}} \in \mathcal{F} L^{p}\left(Q_{1 / \beta}\right)$ then there exists a unique function $m \in L^{p}\left(Q_{1 / \beta}\right)$ such that $\hat{m}(n)=c_{n}$ for every $n$, and the norm on $\mathcal{F} L^{p}\left(Q_{1 / \beta}\right)$ is defined by

$$
\|c\|_{\mathcal{F} L^{p}\left(Q_{1 / \beta}\right)}=\|m\|_{p, Q_{1 / \beta}} .
$$

For $1<p<\infty$, Littlewood-Paley theory can be used to give an equivalent norm for (11), cf. [10, Ch. 7].

Definition 4.1. Let $\alpha, \beta>0$ be given. Then $S_{\tilde{\nu}}^{p, q}=\ell_{\tilde{\nu}}^{q}\left(\mathcal{F} L^{p}\left(Q_{1 / \beta}\right)\right)$ will denote the space of all $\mathcal{F} L^{p}\left(Q_{1 / \beta}\right)$-valued sequences which are $\ell_{\tilde{\nu}^{-}}^{q}$ summable. That is, a doubly-indexed sequence $c=\left(c_{k n}\right)_{k, n \in \mathbb{Z}^{d}}$ lies in $S_{\tilde{\nu}}^{p, q}$ if for each $k \in \mathbb{Z}^{d}$ there exists $m_{k} \in L^{p}\left(Q_{1 / \beta}\right)$ such that

$$
\hat{m}_{k}(n)=c_{k n}, \quad k, n \in \mathbb{Z}^{d}
$$

and such that

$$
\|c\|_{S_{\bar{\nu}}^{p, q}}=\left(\sum_{k \in \mathbb{Z}^{d}}\left\|m_{k}\right\|_{p, Q_{1 / \beta}}^{q} \tilde{\nu}(k)^{q}\right)^{1 / q}<\infty
$$

with the usual change if $q=\infty$.

When $1<p<\infty$, we can write $m_{k}$ as a Fourier series

$$
m_{k}(x)=\sum_{n \in \mathbb{Z}^{d}} c_{k n} e^{2 \pi i \beta n \cdot x}
$$

in the sense that the square partial sums of (12) converge to $m_{k}$ in the norm of $L^{p}\left(Q_{1 / \beta}\right)$, cf. [30], [38]. Hence, for $1<p<\infty$ and $1 \leq q<\infty$ we can write the norm on $S_{\tilde{\nu}}^{p, q}$ as

$$
\|c\|_{S_{\tilde{\nu}}^{p, q}}=\left(\sum_{k \in \mathbb{Z}^{d}}\left(\int_{Q_{1 / \beta}}\left|\sum_{n \in \mathbb{Z}^{d}} c_{k n} e^{2 \pi i \beta n \cdot x}\right|^{p} d x\right)^{q / p} \tilde{\nu}(k)^{q}\right)^{1 / q} .
$$

Note that for $p=2$, we have via the Plancherel theorem that $S_{\tilde{\nu}}^{2, q}=$ $\ell_{\tilde{\nu}}^{2, q}$, However, for general $p, S_{\tilde{\nu}}^{p, q}$ is not a "solid" space. In particular, changing the phases of the $c_{k n}$ can change the norm of $c$. 


\subsection{Boundedness of the analysis and synthesis operators}

Our first main result states some facts which do not require a frame hypothesis. In particular, the following result makes precise the meaning and behavior of the analysis and synthesis operators on the amalgam spaces, and shows that the Walnut representation, which is an extremely useful tool in Gabor analysis, holds on the amalgam spaces.

Theorem 4.2. Let $\nu$ be an $\omega$-moderate weight on $\mathbb{R}^{d}$. Let $\alpha, \beta>0$ and $1 \leq p, q \leq \infty$ be given. Fix $g, \gamma \in W\left(L^{\infty}, L_{\omega}^{1}\right)$. Then the following statements hold.

a. The analysis operator $C_{g} f=\left(\left\langle f, M_{\beta n} T_{\alpha k} g\right\rangle\right)_{k, n \in \mathbb{Z}^{d}}$ is a bounded mapping $C_{g}: W\left(L^{p}, L_{\nu}^{q}\right) \rightarrow S_{\tilde{\nu}}^{p, q}$, Moreover, there exist unique functions $m_{k} \in L^{p}\left(Q_{1 / \beta}\right)$ which satisfy $\hat{m}_{k}(n)=C_{g} f(k, n)$ for all $k, n \in \mathbb{Z}^{d}$, and these are given explicitly by

$$
\begin{aligned}
m_{k}(x) & =\beta^{-d} \sum_{n \in \mathbb{Z}^{d}}\left(f \cdot T_{\alpha k} \bar{g}\right)\left(x-\frac{n}{\beta}\right) \\
& =\beta^{-d} \sum_{n \in \mathbb{Z}^{d}}\left(T_{\frac{n}{\beta}} f \cdot T_{\alpha k+\frac{n}{\beta}} \bar{g}\right)(x) .
\end{aligned}
$$

The series on the right side of (13) converges unconditionally in $L^{p}\left(Q_{1 / \beta}\right)$ (unconditionally in the $\sigma\left(L^{\infty}\left(Q_{1 / \beta}\right), L^{1}\left(Q_{1 / \beta}\right)\right.$ topology if $p=\infty)$.

b. Given $c \in S_{\tilde{\nu}}^{p, q}$, let $m_{k} \in L^{p}\left(Q_{\alpha}\right)$ be the unique functions satisfying $\hat{m}_{k}(n)=c_{k n}$ for all $k, n \in \mathbb{Z}^{d}$. Then the series

$$
R_{g} c=\sum_{k \in \mathbb{Z}^{d}} m_{k} \cdot T_{\alpha k} g
$$

converges unconditionally in $W\left(L^{p}, L_{\nu}^{q}\right)$ (unconditionally in the $\sigma\left(W\left(L^{p}, L_{\nu}^{q}\right), W\left(L^{p^{\prime}}, L_{1 / \nu}^{q^{\prime}}\right)\right)$ topology if $p=\infty$ or $\left.q=\infty\right)$, and $R_{g}$ is a bounded mapping $R_{g}: S_{\tilde{\nu}}^{p, q} \rightarrow W\left(L^{p}, L_{\nu}^{q}\right)$.

c. The Walnut representation

$$
R_{\gamma} C_{g} f=\beta^{-d} \sum_{n \in \mathbb{Z}^{d}} G_{n} \cdot T_{\frac{n}{\beta}} f
$$


holds for $f \in W\left(L^{p}, L_{\nu}^{q}\right)$, with the series on the right of (15) converging absolutely in $W\left(L^{p}, L_{\nu}^{q}\right)$, and where

$$
\begin{aligned}
& G_{n}(x)=\sum_{k \in \mathbb{Z}^{d}} \overline{g\left(x-\frac{n}{\beta}-\alpha k\right)} \gamma(x-\alpha k) \\
& =\sum_{k \in \mathbb{Z}^{d}}\left(T_{\alpha k+\frac{n}{\beta}} \bar{g} \cdot T_{\alpha k} \gamma\right)(x) .
\end{aligned}
$$

Remark 4.3. When $1<p<\infty$, the functions $m_{k}$ appearing in (14) can be written as Fourier series, allowing $R_{g} c$ to be written as the iterated sum

$$
R_{g} c(x)=\sum_{k \in \mathbb{Z}^{d}}\left(\sum_{n \in \mathbb{Z}^{d}} c_{k n} e^{2 \pi i \beta n \cdot x}\right) T_{\alpha k} g(x),
$$

i.e., the same series as appears in (9). When $p=1$ or $p=\infty$, this is not the case. The functions $m_{k}$ are still uniquely determined by $c$, but cannot be written as Fourier series. When $p=q=2$, both the inner and outer sums in the iterated series in (17) converge unconditionally, and then $R_{g} c$ can also be written as the double sum in (8), with unconditional convergence of that series.

\subsection{Gabor expansions in the amalgam spaces}

Under the assumption that $\mathcal{G}(g, \alpha, \beta)$ is a frame for $L^{2}\left(\mathbb{R}^{d}\right)$, we obtain the following result.

Theorem 4.4. Let $\nu$ be an $\omega$-moderate weight on $\mathbb{R}^{d}$, and let $\alpha, \beta>0$ and $1 \leq p, q \leq \infty$ be given. Assume that $g, \gamma \in W\left(L^{\infty}, L_{\omega}^{1}\right)$ are such that $\mathcal{G}(g, \alpha, \beta)$ is a Gabor frame for $L^{2}$ with dual frame $\mathcal{G}(\gamma, \alpha, \beta)$. Then the following statements hold.

a. We have the norm equivalence $\|f\|_{W\left(L^{p}, L_{\nu}^{q}\right)} \asymp\left\|C_{g} f\right\|_{S_{\tilde{\nu}}^{p, q} .}$

b. $R_{\gamma} C_{g}=I$ on $W\left(L^{p}, L_{\nu}^{q}\right)$.

c. A function $f \in W\left(L^{1}, L_{1 / \omega}^{\infty}\right)$ belongs to $W\left(L^{p}, L_{\nu}^{q}\right)$ if and only if $C_{g} f \in S_{\tilde{\nu}}^{p, q}$.

Remark 4.5. a. Theorem 4.4 says that, given an appropriate condition on the window $g$ and its dual window $\gamma$, a Gabor frame for $L^{2}$ extends to the amalgam spaces and provides "Gabor expansions" for the amalgam 
spaces in the sense that we have the factorization of the identity as $I=R_{\gamma} C_{g}$. The specific form of these expansions is that given $f$, there exist functions $m_{k}$ such that $f=R_{\gamma} C_{g} f=\sum m_{k} \cdot T_{\alpha k} g$. When $1<$ $p<\infty$, the functions $m_{k}$ can be realized as Fourier series, leading to an expansion of the form

$$
f(x)=R_{\gamma} C_{g} f(x)=\sum_{k \in \mathbb{Z}^{d}}\left(\sum_{n \in \mathbb{Z}^{d}}\left\langle f, M_{\beta n} T_{\alpha k} \gamma\right\rangle e^{2 \pi i \beta n \cdot x}\right) T_{\alpha k} g(x) .
$$

The inner sum defining $m_{k}$ converges conditionally in general, while the outer sum converges unconditionally.

b. For the case $p=1$, the functions $m_{k}$ cannot be written as Fourier series, so we do not have a series expansion of the form (18). A different approach to the case $p=q=1$ and $\nu=1$, based on Littlewood-Paley theory, is developed by Gilbert and Lakey in [22], where they show that Gabor frames can be used to characterize a Hardy-type space on the line.

c. Theorem 4.4c says that if we use the "largest" amalgam space $W\left(L^{1}, L_{1 / \omega}^{\infty}\right)$ as our "universe," then membership of a function in an amalgam $W\left(L^{p}, L_{\nu}^{q}\right)$ is characterized by membership of its sequence of Gabor coefficients in an appropriate sequence space. By imposing additional restrictions on $g, \gamma$, we could enlarge the universe on which this characterization is valid. In particular, if we required $g, \gamma$ to lie in the Schwartz class $\mathcal{S}$, then the universe on which this characterization was valid would be the space $\mathcal{S}^{\prime}$ of tempered distributions.

d. For the case of the modulation spaces, there is a deep result that states that if $g$ lies in the Feichtinger algebra $M_{\omega}^{1}$, then the dual window $\gamma$ will lie in $M_{\omega}^{1}$ as well, cf. Theorem 3.2. For the case of the amalgam spaces, we do not know if the assumption $g \in W\left(L^{\infty}, L_{\omega}^{1}\right)$ implies that the dual window $\gamma$ also lies in that space. This is an interesting and possibly difficult open question.

\subsection{Convergence of Gabor expansions}

As pointed out above, when $1<p<\infty$, the synthesis operator $R_{g}$ can be written as the iterated sum (17). The inner series in this sum converges conditionally in general, while the outer series converges unconditionally. Our next result shows that this series can also be written as a double sum, as in (8), but because the proof relies on the convergence of Fourier 
series in $L^{p}$, the convergence is conditional in general. In dealing with Fourier series in higher dimensions, it is important to use the maximum norm $|x|=\max \left\{\left|x_{1}\right|, \ldots,\left|x_{d}\right|\right\}$ on $\mathbb{R}^{d}$.

Proposition 4.6. Let $\nu$ be an $\omega$-moderate weight. Let $\alpha, \beta>0$ and $1<p<\infty, 1 \leq q<\infty$ be given. Assume that $g, \gamma \in W\left(L^{\infty}, L_{\omega}^{1}\right)$ are such that $\mathcal{G}(g, \alpha, \beta)$ is a Gabor frame for $L^{2}$ with dual window $\gamma$. Then the following statements hold.

a. If $c \in S_{\tilde{\nu}}^{p, q}$, then the partial sums

$$
S_{K, N} c=\sum_{|k| \leq K} \sum_{|n| \leq N} c_{k n} M_{\beta n} T_{\alpha k} g, \quad K, N>0,
$$

converge to $R_{g} c$ in the norm of $W\left(L^{p}, L_{\nu}^{q}\right)$, i.e., for each $\varepsilon>0$ there exist $K_{0}, N_{0}>0$ such that

$$
\forall K \geq K_{0}, \quad \forall N \geq N_{0}, \quad\left\|R_{g} c-S_{K, N} c\right\|_{W\left(L^{p}, L_{\nu}^{q}\right)}<\varepsilon .
$$

b. If $f \in W\left(L^{p}, L_{\nu}^{q}\right)$, then the partial sums of the Gabor expansion of $f$,

$$
S_{K, N}\left(C_{g} f\right)=\sum_{|k| \leq K} \sum_{|n| \leq N}\left\langle f, M_{\beta n} T_{\alpha k} g\right\rangle M_{\beta n} T_{\alpha k} \gamma,
$$

converge to $f$ in the norm of $W\left(L^{p}, L_{\nu}^{q}\right)$.

\subsection{Necessary conditions on the window}

Our final main result provides a partial converse to Theorem 4.2a. In particular, Theorem 4.2a implies that if $g \in W\left(L^{\infty}, L_{\omega}^{1}\right)$, then $C_{g}$ is bounded on each $W\left(L^{p}, L_{\nu}^{q}\right)$. In the converse direction, if $g$ is a measurable function and $1 \leq p, q \leq \infty$ are given, then in order for $C_{g}$ to be welldefined on $W\left(L^{p}, L_{\nu}^{q}\right)$, we must at least have $C_{g} f(0,0)=\langle f, g\rangle=\int f \bar{g}$ defined for each $f \in W\left(L^{p}, L_{\nu}^{q}\right)$. Hence $f \bar{g} \in L^{1}$ for all such $f$, so we immediately have that $g$ must lie in the Köthe dual of $W\left(L^{p}, L_{\nu}^{q}\right)$, which is $W\left(L^{p^{\prime}}, L_{1 / \nu}^{q^{\prime}}\right)$.

For the unweighted case, we obtain the following further necessary condition in order that $C_{g}$ be bounded on $W\left(L^{p}, L^{\infty}\right)$. For the case $p=2$, this result was obtained by Balan in [5] and published in [6], [7]. Extensions of some other results from [5] to the case $p \neq 2$ are also possible by combining the arguments of Balan with the techniques of this paper. 
Theorem 4.7. Let $\alpha, \beta>0$ and $1<p<\infty$ be given. If $g \in W\left(L^{p^{\prime}}, L^{1}\right)$ and $C_{g}$ is a bounded map from $W\left(L^{p}, L^{\infty}\right)$ to $S^{p, \infty}$, then $g \in W\left(L^{\infty}, L^{p}\right)$.

As noted above, the hypothesis $g \in W\left(L^{p^{\prime}}, L^{1}\right)$ is not a limitation on the generality of the result, as it is necessary in order that $C_{g}$ can even be defined. Furthermore, if $1<p<\infty$ then $W\left(L^{\infty}, L^{p}\right)$ is not contained in $W\left(L^{p^{\prime}}, L^{1}\right)$ nor conversely, so Theorem 4.7 is not a trivial consequence of embeddings of modulation spaces. The result is also true if $p=1$, but in this case $W\left(L^{\infty}, L^{p}\right)=W\left(L^{p^{\prime}}, L^{1}\right)$ and there is no new information gained.

\section{Proofs}

In this section, we present the proofs of the results stated above. First, Section 5.1 provides some useful lemmas. The proof of Theorem 4.2 is divided into Sections 5.2-5.4, which deal respectively with the synthesis operator, the analysis operator, and the Walnut representation. Section 5.5 contains the proof of Theorem 4.4, Section 5.6 contains the proof of Proposition 4.6, and Section 5.7 contains the proof of Theorem 4.7 .

\subsection{Lemmas}

The following lemmas will be important in the sequel. The first lemma is simply a counting argument.

Lemma 5.1. Let $\alpha, \beta>0$ be given. Let $K_{\alpha \beta}$ be the maximum number of $\frac{1}{\beta} \mathbb{Z}^{d}$-translates of $Q_{1 / \beta}$ required to cover any $\alpha \mathbb{Z}^{d}$-translate of $Q_{\alpha}$, i.e.,

$$
K_{\alpha \beta}=\max _{k \in \mathbb{Z}^{d}} \#\left\{\ell \in \mathbb{Z}^{d}:\left|\left(\frac{\ell}{\beta}+Q_{1 / \beta}\right) \cap\left(\alpha k+Q_{\alpha}\right)\right|>0\right\} .
$$

Then given $1 \leq p \leq \infty$, we have for any $1 / \beta$-periodic function $m \in$ $L^{p}\left(Q_{1 / \beta}\right)$ and any $k \in \mathbb{Z}^{d}$ that

$$
\|m\|_{p, \alpha k+Q_{\alpha}} \leq K_{\alpha \beta}^{1 / p}\|m\|_{p, Q_{1 / \beta}}
$$

where $K_{\alpha \beta}^{1 / \infty}=1$.

The second lemma is a weighted version of an estimate that is useful in the Walnut representation of the Gabor frame operator on $L^{2}$, see [34, Lemma 2.2]. 
Lemma 5.2. Let $\omega$ be a submultiplicative weight, and let $\alpha, \beta>0$ be given. Then there exists a constant $C=C(\alpha, \beta, \omega)>0$ such that if $g$, $\gamma \in W\left(L^{\infty}, L_{\omega}^{1}\right)$ and the functions $G_{n}$ are defined by $(16)$, then

$$
\sum_{n \in \mathbb{Z}^{d}}\left\|G_{n}\right\|_{\infty} \omega\left(\frac{n}{\beta}\right) \leq C\|g\|_{W\left(L^{\infty}, L_{\omega}^{1}\right)}\|\gamma\|_{W\left(L^{\infty}, L_{\omega}^{1}\right)} .
$$

Proof. It follows from Lemma 2.1 and the fact that $\omega$ is $\omega$-moderate that $\|f \omega\|_{W\left(L^{\infty}, L^{1}\right)}$ is an equivalent norm for $W\left(L^{\infty}, L_{\omega}^{1}\right)$. In particular, we have $g \omega, \gamma \omega \in W\left(L^{\infty}, L^{1}\right)$, so by [25, Lemma 6.3.1],

$$
\sum_{n \in \mathbb{Z}^{d}}\left\|\tilde{G}_{n}\right\|_{\infty} \leq\left(\frac{1}{\alpha}+1\right)^{d}(2 \beta+1)^{d}\|g \omega\|_{W\left(L^{\infty}, L^{1}\right)}\|\gamma \omega\|_{W\left(L^{\infty}, L^{1}\right)},
$$

where $\tilde{G}_{n}$ is the analogue of $G_{n}$ with $g$ replaced by $|g| \omega$ and $\gamma$ replaced by $|\gamma| \omega$. Hence,

$$
\begin{aligned}
& \sum_{n \in \mathbb{Z}^{d}}\left\|G_{n}\right\|_{\infty} \omega\left(\frac{n}{\beta}\right) \\
& =\sum_{n \in \mathbb{Z}^{d}} \underset{x \in \mathbb{R}^{d}}{\operatorname{esssup}} \mid \sum_{k \in \mathbb{Z}^{d}} \frac{}{g\left(x-\frac{n}{\beta}-\alpha k\right)} \gamma(x-\alpha k) \times \\
& \leq \sum_{n \in \mathbb{Z}^{d}} \operatorname{esssup}_{x \in \mathbb{R}^{d}} \sum_{k \in \mathbb{Z}^{d}}\left|g\left(x-\frac{n}{\beta}-\alpha k\right)\right| \omega\left(x-\frac{n}{\beta}-\alpha k\right) \times \\
& =\sum_{n \in \mathbb{Z}^{d}}\left\|\tilde{G}_{n}\right\|_{\infty} \quad|\gamma(x-\alpha k)| \omega(x-\alpha k) \\
& \leq C\|g\|_{W\left(L^{\infty}, L_{\omega}^{1}\right)}\|\gamma\|_{W\left(L^{\infty}, L_{\omega}^{1}\right)} .
\end{aligned}
$$

Finally, we need an estimate on the effect of translations on the amalgam space norm.

Lemma 5.3. Let $\nu$ be an $\omega$-moderate weight. Then for $1 \leq p, q \leq \infty$, we have for each $f \in W\left(L^{p}, L_{\nu}^{q}\right)$ and $\ell \in \mathbb{Z}^{d}$ that

$$
\left\|T_{\alpha \ell} f\right\|_{W\left(L^{p}, L_{\nu}^{q}\right)} \leq C_{\nu} \omega(\alpha \ell)\|f\|_{W\left(L^{p}, L_{\nu}^{q}\right)} .
$$




\subsection{Proof of Theorem 4.2b: Boundedness of the synthesis operator}

In this subsection we will prove part $\mathrm{b}$ of Theorem 4.2, establishing the boundedness of the synthesis operator on $S_{\tilde{\nu}}^{p, q}$.

We divide into cases. First, we consider the case $1 \leq p, q<\infty$. We are given $c \in S_{\tilde{\nu}}^{p, q}$, and we must prove that the series (14) defining $R_{g} c$ converges unconditionally in the norm of $W\left(L^{p}, L_{\nu}^{q}\right)$, and that $R_{g}$ so defined is a bounded mapping of $W\left(L^{p}, L_{\nu}^{q}\right)$ into $S_{\tilde{\nu}}^{p, q}$. To show the convergence we will make use of Lemma 2.4.

Fix $\varepsilon>0$. Then, by definition of the norm in $S_{\tilde{\nu}}^{p, q}$, we have that $\sum\left\|m_{k}\right\|_{p, Q_{1 / \beta}}^{q} \tilde{\nu}(k)^{q}<\infty$. Hence there exists a finite set $F_{0}$ such that

$$
\forall \text { finite } F \supset F_{0}, \quad \sum_{k \notin F}\left\|m_{k}\right\|_{p, Q_{1 / \beta}}^{q} \tilde{\nu}(k)^{q}<\varepsilon^{q} .
$$

Recall that $1 / \nu$ is an $\omega$-moderate weight, and let $K_{\alpha \beta}$ be the constant appearing in Lemma 5.1. Fix any $h \in W\left(L^{p^{\prime}}, L_{1 / \nu}^{q^{\prime}}\right)$. Then

$$
\begin{aligned}
& \sum_{k \notin F}\left|\left\langle m_{k} \cdot T_{\alpha k} g, h\right\rangle\right| \\
& \leq \sum_{k \notin F} \int_{\mathbb{R}^{d}}\left|m_{k}(x) T_{\alpha k} g(x) h(x)\right| d x \\
& =\sum_{k \notin F} \sum_{n \in \mathbb{Z}^{d}} \int_{Q_{\alpha}}\left|m_{k}(x) T_{\alpha k} g(x) h(x)\right| T_{\alpha n+\alpha k} \chi_{Q_{\alpha}}(x) d x \\
& \leq \sum_{k \notin F} \sum_{n \in \mathbb{Z}^{d}}\left\|T_{\alpha k} g \cdot T_{\alpha n+\alpha k} \chi_{Q_{\alpha}}\right\|_{\infty}\left\|m_{k}\right\|_{p, \alpha n+\alpha k+Q_{\alpha}} \times \\
& \leq \sum_{n \in \mathbb{Z}^{d}}\left\|g \cdot T_{\alpha n} \chi_{Q_{\alpha}}\right\|_{\infty} \times T_{\alpha n+\alpha k} \chi_{Q_{\alpha}} \|_{p^{\prime}} \frac{\nu(\alpha k)}{\nu(\alpha n+\alpha k-\alpha n)} \\
& \sum_{k \notin F} K_{\alpha \beta}^{1 / p}\left\|m_{k}\right\|_{p, Q_{1 / \beta}}\left\|h \cdot T_{\alpha n+\alpha k} \chi_{Q_{\alpha}}\right\|_{p^{\prime}} \frac{C_{\nu} \nu(\alpha k) \omega(\alpha n)}{\nu(\alpha n+\alpha k)} \\
& \leq C_{\nu} K_{\alpha \beta}^{1 / p} \sum_{n \in \mathbb{Z}^{d}}\left\|g \cdot T_{\alpha n} \chi_{Q_{\alpha}}\right\|_{\infty} \omega(\alpha n)\left(\sum_{k \notin F}\left\|m_{k}\right\|_{p, Q_{1 / \beta}}^{q} \nu(\alpha k)^{q}\right)^{1 / q} \times
\end{aligned}
$$




$$
\left(\sum_{k \in \mathbb{Z}^{d}}\left\|h \cdot T_{\alpha n+\alpha k} \chi_{Q_{\alpha}}\right\|_{p^{\prime}}^{q^{\prime}} \frac{1}{\nu(\alpha n+\alpha k)^{q^{\prime}}}\right)^{1 / q^{\prime}} .
$$

Combining (19) and (20), we have that

$$
\sum_{k \notin F}\left|\left\langle m_{k} \cdot T_{\alpha k} g, h\right\rangle\right| \leq \varepsilon C_{\nu} K_{\alpha \beta}^{1 / p}\|g\|_{W\left(L^{\infty}, L_{\omega}^{1}\right)}\|h\|_{W\left(L^{p^{\prime}}, L_{1 / \nu}^{q^{\prime}}\right)} .
$$

Therefore, taking the supremum over all $h$ of unit norm and appealing to Lemma 2.4, we see that $R_{g} c=\sum m_{k} \cdot T_{\alpha k} g$ converges unconditionally. Further, replacing $F$ by $\mathbb{Z}^{d}$ in the calculation in (20) yields

$$
\begin{aligned}
\left|\left\langle R_{g} c, h\right\rangle\right| & \leq \sum_{k \in \mathbb{Z}^{d}}\left|\left\langle m_{k} \cdot T_{\alpha k} g, h\right\rangle\right| \\
& \leq C_{\nu} K_{\alpha \beta}^{1 / p}\|g\|_{W\left(L^{\infty}, L_{\omega}^{1}\right)}\|c\|_{S_{\bar{\nu}}^{p, q}}\|h\|_{W\left(L^{p^{\prime}}, L_{1 / \nu}^{q^{\prime}}\right)} .
\end{aligned}
$$

Since $W\left(L^{p^{\prime}}, L_{1 / \nu}^{q^{\prime}}\right)$ is the dual space of $W\left(L^{p}, L_{\nu}^{q}\right)$, taking the suprema over all $h$ of unit norm in (21) shows that

$$
\begin{aligned}
\left\|R_{g} c\right\|_{W\left(L^{p}, L_{\nu}^{q}\right)} & =\sup \left\{\left|\left\langle R_{g} c, h\right\rangle\right|:\|h\|_{W\left(L^{p^{\prime}}, L_{1 / \nu}^{q^{\prime}}\right)}=1\right\} \\
& \leq C_{\nu} K_{\alpha \beta}^{1 / p}\|g\|_{W\left(L^{\infty}, L_{\omega}^{1}\right)}\|c\|_{S_{\tilde{\nu}}^{p, q},}
\end{aligned}
$$

so $R_{g}$ is bounded. This completes the proof for the case $1 \leq p, q<\infty$.

When $p=\infty$ or $q=\infty$, we make use of the fact that $W\left(L^{p^{\prime}}, L_{1 / \nu}^{q^{\prime}}\right)$ is the Köthe dual of $W\left(L^{p}, L_{\nu}^{q}\right)$. The fact that the series defining $R_{g} c$ converges in the weak topology is given by the same calculations as in (20), (21), and the fact that the Köthe dual is a norm-fundamental subspace of the dual space means that we can again estimate $\left\|R_{g} c\right\|_{S_{\tilde{\nu}}^{p, q}}$ by using (22). Hence $R_{g}$ is bounded, and the proof is complete.

\subsection{Proof of Theorem 4.2a: Boundedness of the analysis operator}

In this subsection we will prove part a of Theorem 4.2, establishing the boundedness of the analysis operator on $W\left(L^{p}, L_{\nu}^{q}\right)$.

We are given that $g \in W\left(L^{\infty}, L_{\omega}^{1}\right)$ and that $1 \leq p, q \leq \infty$. Let $f \in W\left(L^{p}, L_{\nu}^{q}\right)$, which is a subspace of $W\left(L^{1}, L_{1 / \omega}^{\infty}\right)$. First we must 
show that the functions $m_{k}$ given by (13) are well-defined. Since $m_{k}$ is the $1 / \beta$-periodization of the integrable function $f \cdot T_{\alpha k} g$, the series defining $m_{k}$ converges at least in $L^{1}\left(Q_{\alpha}\right)$. To show that the periodization converges unconditionally in $L^{p}\left(Q_{1 / \beta}\right)$ (weakly if $p=\infty$ ) and to derive a useful estimate, fix any $1 / \beta$-periodic function $h \in L^{p^{\prime}}\left(Q_{1 / \beta}\right)$. Then for each fixed $k$, we have

$$
\begin{aligned}
& \int_{Q_{1 / \beta}} \sum_{n \in \mathbb{Z}^{d}}\left|f\left(x-\frac{n}{\beta}\right) T_{\alpha k} g\left(x-\frac{n}{\beta}\right) h(x)\right| d x \\
& =\int_{\mathbb{R}^{d}}\left|f(x) T_{\alpha k} g(x) h(x)\right| d x \\
& =\sum_{n \in \mathbb{Z}^{d}} \int_{Q_{\alpha}}\left|f(x) T_{\alpha k} g(x) h(x)\right| T_{\alpha k+\alpha n} \chi_{Q_{\alpha}}(x) d x \\
& \leq \sum_{n \in \mathbb{Z}^{d}}\left\|T_{\alpha k} g \cdot T_{\alpha k+\alpha n} \chi_{Q_{\alpha}}\right\|_{\infty}\left\|f \cdot T_{\alpha k+\alpha n} \chi_{Q_{\alpha}}\right\|_{p} \times \\
& \leq \sum_{n \in \mathbb{Z}^{d}}\left\|g \cdot T_{\alpha n} \chi_{Q_{\alpha}}\right\|_{\infty}\left\|f \cdot T_{\alpha k+\alpha n} \chi_{Q_{\alpha}}\right\|_{p} K_{\alpha \beta}^{1 / p^{\prime}} \times \\
& \|h\|_{p^{\prime}, Q_{1 / \beta}} \frac{C_{\nu} \nu(\alpha k+\alpha n) \omega(\alpha n)}{\nu(\alpha k)} \\
& =C_{\nu} K_{\alpha \beta}^{1 / p^{\prime}}\|h\|_{p^{\prime}, Q_{1 / \beta}} \frac{1}{\nu(\alpha k)} \sum_{n \in \mathbb{Z}^{d}}\left\|g \cdot T_{\alpha n} \chi_{Q_{\alpha}}\right\|_{\infty} \omega(\alpha n) \times \\
& \left\|f \cdot T_{\alpha k+\alpha n} \chi_{Q_{\alpha}}\right\|_{p} \nu(\alpha k+\alpha n) .
\end{aligned}
$$

This yields the desired convergence, and taking the suprema in (23) over $h$ with unit norm implies the estimate

$$
\begin{gathered}
\left\|m_{k}\right\|_{p, Q_{1 / \beta}} \leq \beta^{-d} C_{\nu} K_{\alpha \beta}^{1 / p^{\prime}} \frac{1}{\nu(\alpha k)} \sum_{n \in \mathbb{Z}^{d}}\left\|g \cdot T_{\alpha n} \chi_{Q_{\alpha}}\right\|_{\infty} \omega(\alpha n) \times \\
\left\|f \cdot T_{\alpha k+\alpha n} \chi_{Q_{\alpha}}\right\|_{p} \nu(\alpha k+\alpha n) .
\end{gathered}
$$

Second, we show that $\hat{m}_{k}(n)$ has the correct form. Since $e^{2 \pi i \beta n \cdot x} \in$ $L^{p^{\prime}}\left(Q_{1 / \beta}\right)$, we have by the weak convergence of the series defining $m_{k}$ 
that

$$
\begin{aligned}
\hat{m}_{k}(n) & =\beta^{d}\left\langle m_{k}, e^{2 \pi i \beta n \cdot x}\right\rangle \\
& =\sum_{\ell \in \mathbb{Z}^{d}} \int_{Q_{1 / \beta}}\left\langle T_{\frac{\ell}{\beta}} f \cdot T_{\alpha k+\frac{\ell}{\beta}} \bar{g}, e^{2 \pi i \beta n \cdot x}\right\rangle \\
& =\sum_{\ell \in \mathbb{Z}^{d}} \int_{Q_{1 / \beta}} f\left(x-\frac{\ell}{\beta}\right) T_{\alpha k} \bar{g}\left(x-\frac{\ell}{\beta}\right) e^{-2 \pi i \beta n \cdot(x-\ell / \beta)} d x \\
& =\int_{\mathbb{R}^{d}}\left(f \cdot T_{\alpha k} \bar{g}\right)(x) e^{-2 \pi i \beta n \cdot x} d x \\
& =\left\langle f, M_{\beta n} T_{\alpha k} g\right\rangle \\
& =C_{g} f(k, n) .
\end{aligned}
$$

Finally, we must show that $C_{g}$ is a bounded mapping of $W\left(L^{p}, L_{\nu}^{q}\right)$ into $S_{\tilde{\nu}}^{p, q}$. Given $f \in W\left(L^{p}, L_{\nu}^{q}\right)$, to show that $C_{g} f \in S_{\tilde{\nu}}^{p, q}$ we must show that the sequence $r$ given by

$$
r(k)=\left\|m_{k}\right\|_{p, Q_{1 / \beta}}, \quad k \in \mathbb{Z}^{d}
$$

lies in $\ell_{\tilde{\nu}}^{q}$. To do this, fix any sequence $a \in \ell_{1 / \tilde{\nu}}^{q^{\prime}}$. Then, using (24), we have

$$
\begin{aligned}
|\langle r, a\rangle| \leq & \sum_{k \in \mathbb{Z}^{d}}\left\|m_{k}\right\|_{p, Q_{1 / \beta}}|a(k)| \\
\leq & \beta^{-d} C_{\nu} K_{\alpha \beta}^{1 / p^{\prime}} \sum_{n \in \mathbb{Z}^{d}}\left\|g \cdot T_{\alpha n} \chi_{Q_{\alpha}}\right\|_{\infty} \omega(\alpha n) \times \\
& \sum_{k \in \mathbb{Z}^{d}}\left\|f \cdot T_{\alpha k+\alpha n} \chi_{Q_{\alpha}}\right\|_{p} \nu(\alpha k+\alpha n)|a(k)| \frac{1}{\nu(\alpha k)} \\
\leq & \beta^{-d} C_{\nu} K_{\alpha \beta}^{1 / p^{\prime}} \sum_{n \in \mathbb{Z}^{d}}\left\|g \cdot T_{\alpha n} \chi_{Q_{\alpha}}\right\|_{\infty} \omega(\alpha n) \times \\
& \left(\sum_{k \in \mathbb{Z}^{d}}\left\|f \cdot T_{\alpha k+\alpha n} \chi_{Q_{\alpha}}\right\|_{p}^{q} \nu(\alpha k+\alpha n)^{q}\right)^{1 / q} \times \\
& \left(\sum_{k \in \mathbb{Z}^{d}}|a(k)|^{q^{\prime}} \frac{1}{\nu(\alpha k)^{q^{\prime}}}\right)^{1 / q^{\prime}}
\end{aligned}
$$




$$
\leq \quad \beta^{-d} C_{\nu} K_{\alpha \beta}^{1 / p^{\prime}}\|g\|_{W\left(L^{\infty}, L_{\omega}^{1}\right)}\|f\|_{W\left(L^{p}, L_{\nu}^{q}\right)}\|a\|_{\ell_{1 / \tilde{\nu}}^{q^{\prime}}} .
$$

Since $\ell_{1 / \tilde{\nu}}^{q^{\prime}}$ equals $\left(\ell_{\tilde{\nu}}^{q}\right)^{*}$ when $q<\infty$ and is a norm-fundamental subspace when $q=\infty$, taking the suprema in (24) over sequences $a$ with unit norm yields the estimate

$$
\left\|C_{g} f\right\|_{S_{\tilde{\nu}}^{p, q}}=\|r\|_{\ell_{\tilde{\nu}}^{p}} \leq \beta^{-d} C_{\nu} K_{\alpha \beta}^{1 / p^{\prime}}\|g\|_{W\left(L^{\infty}, L_{\omega}^{1}\right)}\|f\|_{W\left(L^{p}, L_{\nu}^{q}\right)} .
$$

Hence $C_{g}$ is a bounded mapping of $W\left(L^{p}, L_{\nu}^{q}\right)$ into $S_{\tilde{\nu}}^{p, q}$.

Remark 5.4. For the case $1<p, q<\infty$, the boundedness of $C_{g}$ could also be shown by proving that $C_{g}: W\left(L^{p}, L_{\nu}^{q}\right) \rightarrow S_{\tilde{\nu}}^{p, q}$ is the adjoint of $R_{g}: S_{1 / \tilde{\nu}}^{p^{\prime}, q^{\prime}} \rightarrow W\left(L^{p^{\prime}}, L_{1 / \nu}^{q^{\prime}}\right)$, and then using the reflexivity of the space $W\left(L^{p}, L_{\nu}^{q}\right)$ and the fact that $1 / \nu$ is also $\omega$-moderate.

\subsection{Proof of Theorem 4.2c: The Walnut representation}

In this subsection we will prove part $\mathrm{c}$ of Theorem 4.2, establishing the validity of the Walnut representation of $R_{\gamma} C_{g}$ on $W\left(L^{p}, L_{\nu}^{q}\right)$.

We are given $g, \gamma \in W\left(L^{\infty}, L_{\omega}^{1}\right)$ and $1 \leq p, q \leq \infty$. For this proof, let us use the equivalent norm for $W\left(L^{p}, L_{\nu}^{q}\right)$ obtained by replacing $\alpha$ in $(2)$ by $1 / \beta$. Then by Lemma 5.3 ,

$$
\left\|T_{\frac{n}{\beta}} f\right\|_{W\left(L^{p}, L_{\nu}^{q}\right)} \leq C_{\nu} \omega\left(\frac{n}{\beta}\right)\|f\|_{W\left(L^{p}, L_{\nu}^{q}\right)} .
$$

Therefore, using the autocorrelation functions $G_{n}$ defined in (16), we have for $f \in W\left(L^{p}, L_{\nu}^{q}\right)$ that

$$
\begin{aligned}
& \sum_{n \in \mathbb{Z}^{d}}\left\|G_{n} \cdot T_{\frac{n}{\beta}} f\right\|_{W\left(L^{p}, L_{\nu}^{q}\right)} \\
& \leq \sum_{n \in \mathbb{Z}^{d}}\left\|G_{n}\right\|_{\infty}\left\|T_{\frac{n}{\beta}} f\right\|_{W\left(L^{p}, L_{\nu}^{q}\right)} \\
& \leq C_{\nu}\|f\|_{W\left(L^{p}, L_{\nu}^{q}\right)} \sum_{n \in \mathbb{Z}^{d}}\left\|G_{n}\right\|_{\infty} \omega\left(\frac{n}{\beta}\right) \\
& \leq C C_{\nu}\|f\|_{W\left(L^{p}, L_{\nu}^{q}\right)}\|g\|_{W\left(L^{\infty}, L_{\omega}^{1}\right)}\|\gamma\|_{W\left(L^{\infty}, L_{\omega}^{1}\right)},
\end{aligned}
$$

the last inequality following from Lemma 5.2. Hence the series $\sum G_{n}$. $T_{\frac{n}{\beta}} f$ converges absolutely in $W\left(L^{p}, L_{\nu}^{q}\right)$. 
Now fix $f \in W\left(L^{p}, L_{\nu}^{q}\right)$. Then $C_{g} f \in S_{\widetilde{\nu}}^{p, q}$ by Theorem 4.2a. Letting $m_{k}$ be defined by (13), we have $C_{g} f(k, n)=\hat{m}_{k}(n)$. Further, $R_{\gamma} C_{g} f=$ $\sum m_{k} \cdot T_{\alpha k} \gamma$, this series converging unconditionally if $p, q<\infty$, or unconditionally in the weak topology otherwise. In any case, for $h \in$ $W\left(L^{p^{\prime}}, L_{1 / \tilde{\nu}}^{q^{\prime}}\right)$ we have

$$
\begin{aligned}
\left\langle R_{\gamma} C_{g} f, h\right\rangle & =\sum_{k \in \mathbb{Z}^{d}}\left\langle m_{k} \cdot T_{\alpha k} \gamma, h\right\rangle \\
& =\sum_{k \in \mathbb{Z}^{d}} \int_{\mathbb{R}^{d}} m_{k}(x) T_{\alpha k} \gamma(x) \bar{h}(x) d x \\
& =\beta^{-d} \sum_{k \in \mathbb{Z}^{d}} \int_{\mathbb{R}^{d}} \sum_{n \in \mathbb{Z}^{d}} T_{\frac{n}{\beta}} f(x) T_{\alpha k+\frac{n}{\beta}} \bar{g}(x) T_{\alpha k} \gamma(x) \bar{h}(x) d x \\
& =\beta^{-d} \sum_{n \in \mathbb{Z}^{d}} \int_{\mathbb{R}^{d}} \sum_{k \in \mathbb{Z}^{d}} T_{\frac{n}{\beta}} f(x) T_{\alpha k+\frac{n}{\beta}} \bar{g}(x) T_{\alpha k} \gamma(x) \bar{h}(x) d x \\
& =\beta^{-d} \sum_{n \in \mathbb{Z}^{d}} \int_{\mathbb{R}^{d}} T_{\frac{n}{\beta}} f(x) G_{n}(x) \bar{h}(x) d x . \\
& =\beta^{-d} \sum_{n \in \mathbb{Z}^{d}}\left\langle G_{n} \cdot T_{\frac{n}{\beta}} f, h\right\rangle,
\end{aligned}
$$

from which (15) follows. The interchanges of integration and summation can be justified by Lemma 5.2 and Fubini's Theorem.

\subsection{Proof of Theorem 4.4: Gabor frames in the amalgam spaces}

In this subsection we will prove Theorem 4.4. We are given $g, \gamma \in$ $W\left(L^{\infty}, L_{\omega}^{1}\right)$ such that $\mathcal{G}(g, \alpha, \beta)$ is a Gabor frame for $L^{2}$ and $\gamma$ is the dual window to $g$. By Theorem 4.2, we have that $C_{g}, C_{\gamma}: W\left(L^{p}, L_{\nu}^{q}\right) \rightarrow$ $S_{\tilde{\nu}}^{p, q}$ and $R_{g}, R_{\gamma}: S_{\tilde{\nu}}^{p, q} \rightarrow W\left(L^{p}, L_{\nu}^{q}\right)$ are bounded mappings for each $1 \leq p, q \leq \infty$ and each $\omega$-moderate weight $\nu$. Further, for the case $p=q=2$ and $\nu=1$, the frame hypothesis implies that the identity $R_{\gamma} C_{g}=I$ holds on $L^{2}$, and the definition of $R_{\gamma}$ given in Theorem 3.1 coincides in this case with the definition of $R_{\gamma}$ given in Theorem 4.2. Letting $G_{n}$ be the autocorrelation functions defined in (16), the fact that $R_{\gamma} C_{g}=I$ holds on $L^{2}$ implies by [25, Thm. 7.3.1] that

$$
\beta^{-d} G_{0}=1 \text { a.e. } \quad \text { and } \quad G_{n}=0 \text { a.e. for } n \neq 0 .
$$


Consequently, using the Walnut representation (15) of $R_{\gamma} C_{g}$ on the space $W\left(L^{p}, L_{\nu}^{q}\right)$, we have for $f \in W\left(L^{p}, L_{\nu}^{q}\right)$ that

$$
R_{\gamma} C_{g} f=\beta^{-d} \sum_{n \in \mathbb{Z}^{d}} G_{n} \cdot T_{\frac{n}{\beta}} f=f .
$$

Hence $R_{\gamma} C_{g}=I$ holds on $W\left(L^{p}, L_{\nu}^{q}\right)$ as well. This proves part b of Theorem 4.4.

Next, given $f \in W\left(L^{p}, L_{\nu}^{q}\right)$, we have

$$
\begin{aligned}
\|f\|_{W\left(L^{p}, L_{\nu}^{q}\right)} & =\left\|R_{\gamma} C_{g} f\right\|_{W\left(L^{p}, L_{\nu}^{q}\right)} \\
& \leq\left\|R_{\gamma}\right\|\left\|C_{g} f\right\|_{S_{\tilde{\nu}}^{p, q}} \\
& \leq\left\|R_{\gamma}\right\|\left\|C_{g}\right\|\|f\|_{W\left(L^{p}, L_{\nu}^{q}\right)} .
\end{aligned}
$$

Consequently, $\left\|C_{g} f\right\|_{S_{\tilde{\nu}}^{p}, q} \asymp\|f\|_{W\left(L^{p}, L_{\nu}^{q}\right)}$, which proves part a of Theorem 4.4 .

Finally, we prove part c of Theorem 4.4. Let $f \in W\left(L^{1}, L_{1 / \omega}^{\infty}\right)$ be given. We must show that $f \in W\left(L^{p}, L_{\nu}^{q}\right)$ if and only if $C_{g} f \in S_{\tilde{\nu}}^{p, q}$. The forward direction, that if $f \in W\left(L^{p}, L_{\nu}^{q}\right)$ then $C_{g} f \in S_{\tilde{\nu}}^{p, q}$, is simply Theorem 4.2a. For the reverse direction, assume that $C_{g} f \in S_{\tilde{\nu}}^{p, q}$. Then by Theorem $4.2 \mathrm{a}$, the function $\tilde{f}=R_{\gamma}\left(C_{g} f\right)$ lies in $W\left(L^{p}, L_{\nu}^{q}\right)$. However, the factorization $R_{\gamma} C_{g}=I$ holds on every amalgam space, including $W\left(L^{1}, L_{1 / \omega}^{\infty}\right)$ in particular, so we also know that $f=R_{\gamma} C_{g} f$. Thus $f=\tilde{f} \in W\left(L^{p}, L_{\nu}^{q}\right)$, which completes the proof.

\subsection{Proof of Proposition 4.6: Convergence of Gabor ex- pansions}

In this subsection we will prove Proposition 4.6. We are given $g, \gamma \in$ $W\left(L^{\infty}, L_{\omega}^{1}\right)$ such that $\mathcal{G}(g, \alpha, \beta)$ is a Gabor frame for $L^{2}$ and $\gamma$ is the dual window to $g$, and we fix $1<p<\infty$ and $1 \leq q<\infty$.

Assume that $c \in S_{\tilde{\nu}}^{p, q}$, and let $m_{k}$ be defined by (13). For $N>0$, write

$$
S_{N} m_{k}=\sum_{|n| \leq N} c_{k n} e^{2 \pi i \beta n \cdot x}
$$

for the partial sums of the Fourier series of $m_{k}$. The exponentials $\left\{e^{2 \pi i \beta n \cdot x}\right\}_{n \in \mathbb{Z}^{d}}$ form a basis for $L^{p}\left(Q_{1 / \beta}\right)$ [30], [38], so, letting $C_{1}$ denote the basis constant for this system, we have for each $k \in \mathbb{Z}^{d}$ that

$$
\lim _{N \rightarrow \infty}\left\|m_{k}-S_{N} m_{k}\right\|_{p, Q_{1 / \beta}}=0
$$


and

$$
\sup _{N>0}\left\|S_{N} m_{k}\right\|_{p, Q_{1 / \beta}} \leq C_{1}\left\|m_{k}\right\|_{p, Q_{1 / \beta}} .
$$

Since $c \in S_{\widetilde{\nu}}^{p, q}$, given $\varepsilon>0$, we can find $K_{0}>0$ such that

$$
\forall K \geq K_{0}, \quad\left(\sum_{|k| \geq K}\left\|m_{k}\right\|_{p, Q_{1 / \beta}}^{q} \tilde{\nu}(k)^{q}\right)^{1 / q}<\varepsilon .
$$

Because of (25) and the fact that $K_{0}$ is finite, we can find an $N_{0}>0$ such that

$$
\forall N \geq N_{0}, \quad \sup _{|k| \leq K_{0}}\left\|m_{k}-S_{N} m_{k}\right\|_{p, Q_{1 / \beta}} \tilde{\nu}(k)<\frac{\varepsilon}{\left(2 K_{0}+1\right)^{d / q}} .
$$

Now, since $c \in S_{\tilde{\nu}}^{p, q}$ and $1<p<\infty$, we know that $R_{g} c$ can be written as the iterated series (17). Write the partial sums of the outer series as

$$
S_{K, \infty} c=\sum_{|k| \leq K}\left(\sum_{n \in \mathbb{Z}^{d}} c_{k n} e^{2 \pi i \beta n \cdot x}\right) T_{\alpha k} g=\sum_{|k| \leq K} m_{k} \cdot T_{\alpha k} g .
$$

Given $K \geq K_{0}$ and $N \geq N_{0}$, write

$$
R_{g} c-S_{K, N} c=\left(R_{g} c-S_{K_{0}, \infty} c\right)+\left(S_{K_{0}, \infty} c-S_{K_{0}, N} c\right)+\left(S_{K_{0}, N} c-S_{K, N} c\right) .
$$

We will calculate the $W\left(L^{p}, L_{\nu}^{q}\right)$ norm of each of these terms separately.

For the first term, define a sequence $r$ by $r_{k n}=c_{k n}$ for $|k| \leq K_{0}$ and $n \in \mathbb{Z}^{d}$, and $r_{k n}=0$ otherwise. Then $S_{K_{0}, \infty} c=R_{g} r$, and $R_{g}$ is a bounded mapping of $S_{\tilde{\nu}}^{p, q} \rightarrow W\left(L^{p}, L_{\nu}^{q}\right)$, so using (27) we have

$$
\begin{aligned}
\left\|R_{g} c-S_{K_{0}, \infty} c\right\|_{W\left(L^{p}, L_{\nu}^{q}\right)} & =\left\|R_{g}(c-r)\right\|_{W\left(L^{p}, L_{\nu}^{q}\right)} \\
& \leq\left\|R_{g}\right\|\|c-r\|_{S_{\bar{\nu}}^{p, q}} \\
& =\left\|R_{g}\right\|\left(\sum_{|k|>K_{0}}\left\|m_{k}\right\|_{p, Q_{1 / \beta}}^{q} \tilde{\nu}(k)^{q}\right)^{1 / q} \\
& \leq\left\|R_{g}\right\| \varepsilon .
\end{aligned}
$$

For the second term, define $s_{k n}=c_{k n}$ for $|k| \leq K_{0}$ and $|n| \leq N$, and $s_{k n}=0$ otherwise. Then $S_{K_{0}, N} c=R_{g} s$, so using (28), we have

$$
\left\|S_{K_{0}, \infty} c-S_{K_{0}, N} c\right\|_{W\left(L^{p}, L_{\nu}^{q}\right)}
$$




$$
\begin{aligned}
& \leq\left\|R_{g}\right\|\|r-s\|_{S_{\tilde{\nu}}^{p, q}} \\
& =\left\|R_{g}\right\|\left(\sum_{|k| \leq K_{0}}\left\|m_{k}-S_{N} m_{k}\right\|_{p, Q_{1 / \beta}}^{q} \tilde{\nu}(k)^{q}\right)^{1 / q} \\
& \leq\left\|R_{g}\right\| \varepsilon .
\end{aligned}
$$

For the third term, define $t_{k n}=c_{k n}$ for $|k| \leq K$ and $|n| \leq N$, and $t_{k n}=0$ otherwise. Then $S_{K_{0}, N} c=R_{g} t$, so using (26) and (27), we have

$$
\begin{aligned}
& \left\|S_{K_{0}, N} c-S_{K, N} c\right\|_{W\left(L^{p}, L_{\nu}^{q}\right)} \\
& \leq\left\|R_{g}\right\|\|s-t\|_{S_{\nu}^{p, q}} \\
& =\left\|R_{g}\right\|\left(\sum_{K_{0}<|k| \leq K}\left\|S_{N} m_{k}\right\|_{p, Q_{1 / \beta}}^{q} \tilde{\nu}(k)^{q}\right)^{1 / q} \\
& \leq C_{1}\left\|R_{g}\right\|\left(\sum_{K_{0}<|k| \leq K}\left\|m_{k}\right\|_{p, Q_{1 / \beta}}^{q} \tilde{\nu}(k)^{q}\right)^{1 / q} \\
& \leq C_{1}\left\|R_{g}\right\| \varepsilon .
\end{aligned}
$$

Applying (30)-(32) to (29), we see that $\left\|R_{g} c-S_{K, N} c\right\|_{W\left(L^{p}, L_{\nu}^{q}\right)} \leq$ $\left(2+C_{1}\right)\left\|R_{g}\right\| \varepsilon$, which completes the proof.

\subsection{Proof of Proposition 4.7: Necessary conditions}

We will prove Theorem 4.7 in this subsection. We assume that $g \in$ $W\left(L^{p^{\prime}}, L^{1}\right)$ is such that $C_{g}$ is a bounded map from $W\left(L^{p}, L^{\infty}\right)$ to $S^{p, \infty}$, where $1<p<\infty$, and we wish to show that $g \in W\left(L^{\infty}, L^{p}\right)$. Let us show first that $g \in L^{\infty}$. If not, then given any $D>0$ there would exist a set $J$ contained in some cube $\frac{\ell}{\beta}+Q_{1 / \beta}$ and with positive measure such that $|g(x)|>D$ on $J$.

Set $f=\frac{1}{|J|^{1 / p}} e^{i \arg g} \chi_{J}$. Using the equivalent norm for $W\left(L^{p}, L^{\infty}\right)$ obtained by replacing $\alpha$ in $(2)$ by $1 / \beta$, we have that $\|f\|_{W\left(L^{p}, L^{\infty}\right)} \leq 1$. By hypothesis, $C_{g} f \in S^{p, \infty}$, so there exist $1 / \beta$-periodic functions $m_{k}$ such that $\hat{m}_{k}(n)=C_{g} f(k, n)$. Since $f \cdot T_{\alpha k} \bar{g} \in L^{1}$, it is easy to see that $m_{k}$ is given by (13). In particular, considering $k=0$ we have

$$
\left\|m_{0}\right\|_{p, Q_{1 / \beta}}^{p}=\beta^{-p d} \int_{Q_{1 / \beta}}\left|\sum_{n \in \mathbb{Z}^{d}} f\left(x-\frac{n}{\beta}\right) \bar{g}\left(x-\frac{n}{\beta}\right)\right|^{p} d x
$$




$$
\begin{aligned}
& =\frac{\beta^{-p d}}{|J|} \int_{\frac{\ell}{\beta}+Q_{1 / \beta}} \chi_{J}(x)|g(x)|^{p} d x \\
& \geq \beta^{-p d} D^{p} .
\end{aligned}
$$

Hence

$$
\begin{aligned}
D & \leq \beta^{d} \sup _{k \in \mathbb{Z}^{d}}\left\|m_{k}\right\|_{p, Q_{1 / \beta}} \\
& =\beta^{d}\left\|C_{g} f\right\|_{S^{p, \infty}} \\
& \leq \beta^{d}\left\|C_{g}\right\|\|f\|_{W\left(L^{p}, L^{\infty}\right)} \leq \beta^{d}\left\|C_{g}\right\| .
\end{aligned}
$$

But since $D$ is arbitrary, this contradicts the fact that $C_{g}$ is a bounded mapping. Hence $g$ must be in $L^{\infty}$.

Now we show that $g \in W\left(L^{\infty}, L^{p}\right)$. Fix $\varepsilon>0$, and for each $n \in \mathbb{Z}^{d}$ define

$$
J_{n}=\left\{x \in \frac{n}{\beta}+Q_{1 / \beta}:|g(x)| \geq \frac{1}{2}\|g\|_{\infty, \frac{n}{\beta}+Q_{1 / \beta}}\right\} .
$$

Then set $J_{n}^{\prime}=J_{n}$ if $\left|J_{n}\right| \leq \varepsilon$, otherwise let $J_{n}^{\prime}$ be a subset of $J_{n}$ of measure $\varepsilon$. Let

$$
N_{\varepsilon}=\sup \left\{N \in \mathbb{N}:\left|J_{n}^{\prime}\right| \geq \frac{\varepsilon}{2} \text { for all }|n| \leq N\right\}
$$

Note that $N_{\varepsilon} \rightarrow \infty$ as $\varepsilon \rightarrow 0$ (and may even be $\infty$ for some $\varepsilon$ ). Define $f=e^{i \arg g} \sum_{|n| \leq N_{\varepsilon}} \chi_{J_{n}^{\prime}}$, and note that $\|f\|_{W\left(L^{p}, L^{\infty}\right)} \leq \varepsilon^{1 / p}$. Therefore $C_{g} f \in S^{p, \infty}$, and letting $m_{k}$ be defined by (13), we have

$$
\begin{aligned}
\left\|m_{0}\right\|_{p, Q_{1 / \beta}}^{p} & =\beta^{-p d} \sum_{|n| \leq N_{\varepsilon}} \int_{\frac{n}{\beta}+Q_{1 / \beta}}|g(x)|^{p} \chi_{J_{n}^{\prime}}(x) d x \\
& \geq \beta^{-p d} \sum_{|n| \leq N_{\varepsilon}}\left(\frac{\left\|g \cdot T_{\frac{n}{\beta}} \chi_{Q_{1 / \beta}}\right\|_{\infty}}{2}\right)^{p}\left|J_{n}^{\prime}\right| \\
& \geq \beta^{-p d} 2^{-p-1} \varepsilon \sum_{|n| \leq N_{\varepsilon}}\left\|g \cdot T_{\frac{n}{\beta}} \chi_{Q_{1 / \beta}}\right\|_{\infty}^{p} .
\end{aligned}
$$

Hence

$$
\sum_{|n| \leq N_{\varepsilon}}\left\|g \cdot T_{\frac{n}{\beta}} \chi_{Q_{1 / \beta}}\right\|_{\infty}^{p} \leq \frac{\beta^{p d} 2^{p+1}}{\varepsilon} \sup _{k \in \mathbb{Z}^{d}}\left\|m_{k}\right\|_{p, Q_{1 / \beta}}^{p}
$$




$$
\begin{aligned}
& =\frac{\beta^{p d} 2^{p+1}}{\varepsilon}\left\|C_{g} f\right\|_{S^{p, \infty}}^{p} \\
& \leq \frac{\beta^{p d} 2^{p+1}}{\varepsilon}\left\|C_{g}\right\|^{p}\|f\|_{W\left(L^{p}, L^{\infty}\right)}^{p} \\
& \leq \beta^{p d} 2^{p+1}\left\|C_{g}\right\|^{p} .
\end{aligned}
$$

Since $N_{\varepsilon} \rightarrow \infty$ as $\varepsilon \rightarrow 0$, this implies that $g \in W\left(L^{\infty}, L^{p}\right)$.

\section{Acknowledgments}

We are deeply indebted to Radu Balan for valuable discussions concerning necessary conditions on the window.

C. Heil and K. Okoudjou were partially supported by NSF Grants DMS-9970524 and DMS-0139261. K. Gröchenig was partially supported by FWF project P-14485-MAT.

\section{References}

[1] A. Aldroubi, Non-uniform weighted average sampling and exact reconstruction, preprint, 2001.

[2] A. Aldroubi and H. Feichtinger, Exact iterative reconstruction algorithm for multivariate irregularly sampled functions in spline-like spaces: the $L_{p}$ theory, Proc. Amer. Math. Soc., 126, 2677-2686, 1998.

[3] A. Aldroubi and K. Gröchenig, Beurling-Landau-type theorems for non-uniform sampling in shift invariant spaces, J. Fourier Anal. Appl., 6, 91-101, 2000.

[4] A. Aldroubi and K. Gröchenig, Non-uniform sampling and reconstruction in shift-invariant spaces, SIAM Rev., 43, 585-620, 2001.

[5] R. V. Balan, A Study of Weyl-Heisenberg and Wavelet Frames, Ph.D. Thesis, Princeton University, 1998.

[6] R. Balan, I. Daubechies, and V. Vaishampayan, The analysis and design of windowed Fourier frame based multiple description source coding schemes, IEEE Trans. Inform. Theory, 46, 2491-2536, 2000. 
[7] R. Balan and I. Daubechies, Optimal stochastic approximations and encoding schemes using Weyl-Heisenberg sets, in: Advances in Gabor Analysis, H. G. Feichtinger and T. Strohmer, eds., Birkhäuser, Boston, 259-320, 2003.

[8] C. Bennett and R. Sharpley, Interpolation of Operators, Academic Press, Boston, 1988.

[9] I. Daubechies, Ten Lectures on Wavelets, SIAM, Philadelphia, 1992.

[10] R. E. Edwards and G. I. Gaudry, Littlewood-Paley and Multiplier Theory, Springer-Verlag, Berlin-Heidelberg-New York, 1977.

[11] H. G. Feichtinger, Gewichtsfunktionen auf lokalkompakten Gruppen, Sitzber.d. österr. Adad. Wiss., 188, 451-471, 1979.

[12] H. G. Feichtinger, Banach convolution algebras of Wiener type, in: Functions, Series, Operators, Proc. Conf. Budapest 35, Colloq. Math. Soc. János Bolyai, B. Sz.-Nagy and J. Szabados, eds., North Holland, Amsterdam, 509-524, 1983.

[13] H. G. Feichtinger, Banach spaces of distributions of Wiener's type and interpolation, in: "Functional Analysis and Approximation" (Oberwolfach, 1980), Internat. Ser. Numer. Math., 60, Birkhäuser, Basel, 153-165, 1981.

[14] H. G. Feichtinger, Wiener amalgams over Euclidean spaces and some of their applications, in: Function spaces (Edwardsville, IL, 1990), Lecture Notes in Pure and Appl. Math. 136, K. Jarosz, ed., Dekker, New York, 123-137, 1992.

[15] H. G. Feichtinger, New results on regular and irregular sampling based on Wiener amalgams, in: Function spaces (Edwardsville, IL, 1990), Lecture Notes in Pure and Appl. Math. 136, K. Jarosz, ed., Dekker, New York, 107-121, 1992.

[16] H. G. Feichtinger, Amalgam spaces and generalized harmonic analysis, in: Proceedings of the Norbert Wiener Centenary Congress, 1994 (East Lansing, MI, 1994), Proc. Sympos. Appl. Math., 52, V. Mendrekar et al., eds., Amer. Math. Soc., Providence, RI, 141150, 1997. 
[17] H. G. Feichtinger and K. Gröchenig, Gabor frames and timefrequency analysis of distributions, J. Funct. Anal., 146, 464-495, 1997.

[18] H. G. Feichtinger, K. Gröchenig, and D. Walnut, Wilson bases and modulation spaces, Math. Nachr., 155, 7-17, 1992.

[19] H. G. Feichtinger and T. Werther, Robustness of regular sampling in Sobolev algebras, in: Sampling, Wavelets and Tomography, J. J. Benedetto and A. Zayed, eds., Birkhäuser, Boston, to appear.

[20] G. B. Folland, Harmonic Analysis on Phase Space, Ann. of Math. Studies, Princeton University Press, Princeton, NJ, 1989.

[21] J. J. F. Fournier and J. Stewart, Amalgams of $L^{p}$ and $\ell^{q}$, Bull. Amer. Math. Soc. (N.S.), 13, 1-21, 1985.

[22] J. E. Gilbert and J. D. Lakey, On the characterization of the local Hardy space by Gabor frames, preprint, 2000.

[23] L. Grafakos and C. Lennard, Characterization of $L^{p}\left(\mathbb{R}^{n}\right)$ using Gabor frames, J. Fourier Anal. Appl., 7, 101-126, 2001.

[24] K. Gröchenig, Describing functions: Atomic decompositions versus frames, Monatshefte für Mathematik, 112, 1-41, 1991.

[25] K. Gröchenig, Foundations of Time-Frequency Analysis, Birkhäuser, Boston, 2001.

[26] K. Gröchenig and C. Heil, Gabor meets Littlewood-Paley: Gabor expansions in $L^{p}\left(\mathbb{R}^{d}\right)$, Studia Math., 146, 15-33, 2001.

[27] K. Gröchenig and M. Leinert, Wiener's lemma for twisted convolution and Gabor frames, preprint, 2002.

[28] C. Heil, An introduction to weighted Wiener amalgams, in: Proc. International Conference on Wavelets and their Applications (Chennai, January 2002), R. Ramakrishnan and S. Thangavelu, eds., to appear.

[29] C. E. Heil and D. F. Walnut, Continuous and discrete wavelet transforms, SIAM Review, 31, 628-666, 1989. 
[30] Y. Katznelson, An Introduction to Harmonic Analysis, Dover, New York, 1976.

[31] Y. Meyer, Wavelets and Operators, Cambridge University Press, Cambridge, 1992.

[32] H. Reiter and J. Stegeman, Classical Harmonic Analysis and Locally Compact Groups, Second Edition, London Mathematical Society Monographs, New Series 22, The Clarendon Press, Oxford University Press, New York, 2000.

[33] I. Singer, Bases in Banach Spaces, I, Springer-Verlag, New York, 1970.

[34] D. Walnut, Continuity properties of the Gabor frame operator, J. Math. Anal. Appl., 165, 479-504, 1992.

[35] N. Wiener, On the representation of functions by trigonometric integrals, Math. Z., 24, 575-616, 1926.

[36] N. Wiener, Tauberian theorems, Ann. of Math., 33, 1-100, 1932.

[37] N. Wiener, The Fourier Integral and Certain of its Applications, MIT Press, Cambridge, 1933.

[38] A. Zygmund, Trigonometric Series, Cambridge University Press, Cambridge, 1959. 